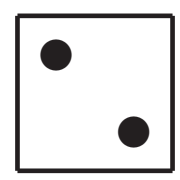

\title{
Quantum Sensing and Metrology
}

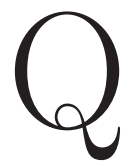

UANTUM sensing is the most exciting quantum technology and it has the most potential to change our lives in the next decade and beyond. Quantum sensors will offer new capabilities with benefits for medicine, defense, intelligence, extractive industries, and many others. Quantum sensing is a precursor technology to quantum computing and communications. In quantum computing, quantum sensors are the literal devices that get information out of a quantum computer, while in quantum communications, quantum sensors are the devices that recover the stream of encryption bits. Thus, quantum sensing will advance as governments pour money into quantum computing and communications.

Quantum sensors use quantum properties and effects to measure or sense physical things. ${ }^{1}$ Sensors based on quantum properties must be constructed such that they are sensitive to the smallest perturbations. This is because the smallest perturbations of the universe necessarily take place at the atomic and subatomic levels - and as such, the only way to measure them is with quantum devices.

In a functioning quantum computer, those perturbations can cause decoherence and thus limit the complexity of programs that the computer can run. In quantum communications, decoherence means that photons traveling down a long fiber-optic cable or through the atmosphere interact with the surrounding medium, losing their in-

${ }^{1}$ This book refers to metrology (that is, measurement, not the study of weather) and sensing under the common label quantum sensing. 
tegrity. This integrity loss imposes limits on the length of a quantum link and the size of quantum networks.

Quantum sensing flips our vantage point. In the quantum sensing context, the exquisite sensitivity of quantum systems is a strength. ${ }^{2}$ Quantum sensors harness this sensitivity of individual quantum particles to measure extraordinarily subtle phenomena.

Atomic clocks, nuclear magnetic resonance (NMR), and magnetic resonance imaging (MRI) are all decades-old forms of quantum sensing. These first-generation quantum sensing systems used classical physics and electronics to make precise measurements of quantum phenomena. Newer second-generation forms of quantum sensing rely on the quantum effects discussed in Appendix B.4, particularly quantum entanglement and superposition. We examine two specific applications of second-generation sensors: signals intelligence (SIGINT), which focuses on communications systems, and in measurement and signature intelligence (MASINT), which focuses on the physical attributes of targets.

We believe quantum sensing is the most consequential technology for our lifetimes because:

- Quantum sensing is the most mature quantum technology and some quantum sensors are already commercialized. The market is likely to grow. That's because sensing is simpler than quantum computing, and because many large, mature industries, such as healthcare, mining, and construction, can directly benefit from measurements that are both more accurate and more precise.

- Quantum sensing has applications in military, intelligence, and law enforcement. Nations with outer space programs have a wider range of quantum sensing options than nations limited to terrestrial applications.

- Some applications of quantum sensing are "stealthy," that is, one may be able to use quantum sensing without being detected.

- Advances in lasers - such as increased power, efficiency, and stability - make some kinds of quantum sensors more powerful

${ }^{2}$ Degen, Reinhard, and Cappellaro, "Quantum Sensing" (2017). 


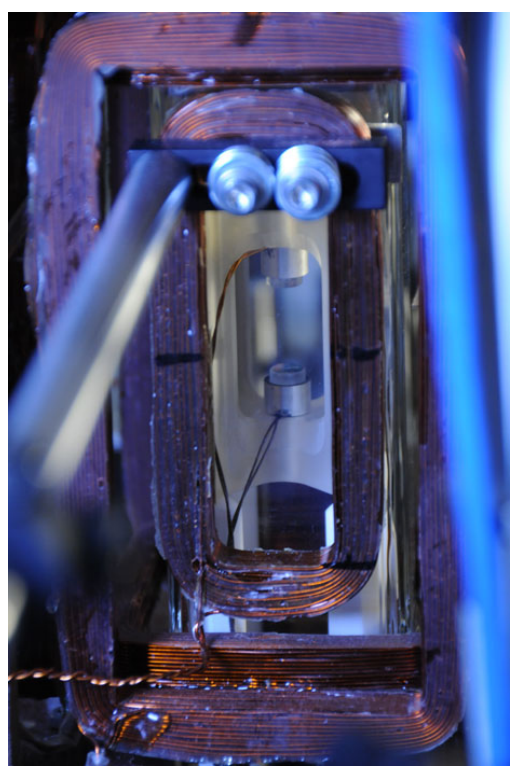

Figure 2.1. Improvement in laser technology is one factor contributing to more exquisite quantum metrology and sensing. Laser stability sets limits on precision, and is affected by the most subtle factors, such as the vibrations caused by photons striking mirrors inside the laser. To address instability, scientists at JILA, a joint University of Colorado/NIST research center, developed a "superradiant" laser. Based on a 1953 hypothesis by R. H. Dicke, the JILA laser traps rubidium atoms between mirrors separated by $2 \mathrm{~cm}$ - the small disks in the center of the photograph. By manipulating the rubidium transitions, the atoms themselves emit a dim, yet coherent laser. In doing so, the rubidium atoms produce light while avoiding the normal, noisy process of synchronizing large numbers of photons. Image public domain by Burrus/NIST.

by increasing their range, their resolution, or the speed with which a measurement can be taken.

- Advances in measuring time precisely using quantum technologies have knock-on effects for the precision of all other kinds of sensing, including location and image resolution.

- Finally, some quantum sensing methods do not require supercooling down to liquid helium temperatures, making them easier to work with and miniaturize. For example, such sensors might be readily made portable - or even used inside the body. 


\section{-Precision, Accuracy, and Repeatability}

Precision, accuracy, and repeatability are three complementary ways to evaluate the performance of a measuring device. For example, imagine you are tracking the height of a child by marking a door-frame with a pencil, and measuring the height of the mark with a yardstick:

precision The word precision means the ability of the measuring device to distinguish two numbers: for example, it may be difficult to tell in the example above if the child is 48 inches or 48.05 inches tall. That's because the precision of a yardstick is roughly $\frac{1}{16}$ inch. With most digital meters, the precision is typically the significance of the rightmost digit.

accuracy This word refers to the difference between the result that you might get using the calculation method described above and the true number. For example, you might report the child as being between 0 inches and 1000 inches tall, which is accurate but not very precise. On the other hand, you might say that the child is 978.01 inches tall. This is precise, but it is unlikely to be accurate. In our example most yardsticks are reasonably accurate unless they are damaged: if your yardstick is missing its first inch, it will be just as precise, but it will be significantly less accurate.

repeatability Something is repeatable if the same answer is obtained by following the same sequence of operations. If the child is fidgeting, it might be very difficult to get a repeatable measurement.

It is possible for a measuring method to be repeatable and precise without being accurate: we might consistently measure the child as being 978.01 inches tall. It is also possible to be accurate without being repeatable or precise: we might measure the child as being between 0 inches and 500 inches, and then as being between 50 and 600 inches. However, it is generally not enough for a measurement to be accurate and precise without it also being repeatable. 


\section{Defining and Redefining The Second}

The second was traditionally defined as being $\frac{1}{86400}$ of a day, each day being divided into 24 hours, each hour divided into 60 minutes, each minute into 60 seconds $(24 \times 60 \times 60=86400)$. But how should it actually be measured?

Several proposals for using the swing of precise pendulums as the standard measure for time started in the seventeenth century. Measurements of such pendulums resulted in the discovery that the earth's gravity is not constant, a result of the planet's bulge and the unequal distribution of minerals beneath the surface.

In the 1930s astronomers discovered that the Earth's rotation is not constant either, because the Earth's atmosphere and water do not turn lockstep with the planet. Instead, the Earth is slowing down at a rate of roughly 2.5 milliseconds per century, which means that each day is imperceptibly longer than the previous.

For the next three decades physicists and astronomers argued which discipline should standardize time. Physicists proposed using the vibration or resonance of a crystal, molecule, or atom, while astronomers proposed using a readily observable periodic motion, such as the rotation of the Moon around the Earth.

The physicists ultimately won, and the second was redefined on October 13, 1967 by the General Conference on Weights and Measures (Conférence Générale des Poids et Mesures, CGPM) to be exactly "the duration of 9192631770 periods of the radiation corresponding to the transition between the two hyperfine levels of the ground state of cesium-133 atom." $a$

It may be more correct to say that the result of the discussions was a truce, however. (These days both physicists and astronomers keep track of the time and the two are synchronized by the addition or subtraction of leap seconds.)

${ }^{a}$ Bureau International des Poids et Mesures, "50th Anniversary of The Adoption of The Atomic Definition of The Second" (2017); Weyers, "Unit of Time Working Group 4.41" (2020). 


\subsection{First-Generation Quantum Sensing}

"First-generation quantum sensors" use classical physics and electronics to observe quantum phenomena.

A familiar technology, the atomic clock, invented in 1959, is based on a quantum hyperfine transition that occurs within a cesium-133 atom. The transition happens when the atom absorbs and then reemits a photon with a frequency of exactly $9.192631770 \mathrm{GHz}$, which is in the microwave frequency range. Modern atomic clocks use a tube of cesium atoms suspended in a vacuum and cooled to nearly absolute zero, to minimize the impact of external forces on each atom's electrons. The clock then adjusts the frequency of the microwave beam until it resonates with the cesium atoms. Once the resonance is achieved, the circuit keeps the frequency locked in place. In 1967 the second was defined in terms of the atomic clock (see the sidebar "Defining and Redefining the Second" on page 35), which measures time by simply counting the number of cycles that elapse: every 9192631770 cycles is precisely one second. Arias and Petit note that the official definition "refers, without saying[,] to 'unperturbed' atoms, that is, those at rest, at zero magnetic and electric fields," reminding us that it is one thing to define a standard in terms of a quantum property and another thing to measure that property with accuracy and precision. ${ }^{3}$

In 1997 physicists clarified that the 1967 definition also required that the cesium atom be at the temperature of absolute zero. Cooling to near absolute zero is performed using lasers. This technique was developed by Steven Chu, Claude Cohen-Tannoudji, and William D. Philips, who received the Nobel Prize in Physics that same year, "for development of methods to cool and trap atoms with laser light."

Quartz watches and computers use a similar approach, although they typically measure the vibrations electronically stimulated in quartz crystals using the piezoelectric effect. Such crystals are easily packaged and are inside most computers, cell phones, and digital watches. Unlike individual atoms of cesium near absolute zero, which absorb energy at a precise frequency dictated by quantum physics, the vibrational frequency of a stimulated quartz crystal can be tuned by altering the crystal's thickness and shape when it is cut. To make

${ }^{3}$ Arias and Petit, "The Hyperfine Transition for The Definition of The Second" (2019). 


\section{Ethanol}<smiles>CCO</smiles>

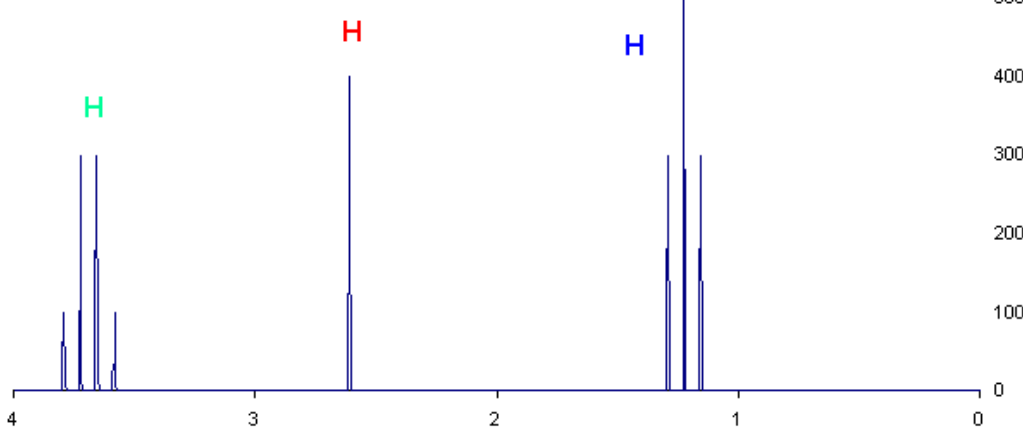

Figure 2.2. The nuclear magnetic resonance spectrum of an ethanol molecule shows that hydrogen atoms exist in one of three configurations. From Wikipedia, by T. Vanschaik. Used under CC-A-SA 3.0.

the engineering easier (and cheaper), the quartz crystals in most digital watches are tuned to vibrate at roughly 32768 times per second.

Another familiar technology that leverages principles of quantum phenomena is Magnetic Resonance Imaging (MRI). MRI creates images of body parts by detecting the magnetic spin of hydrogen. ${ }^{4}$ MRI is based on a molecular property called Nuclear Magnetic Resonance (NMR), in which molecules resonate with a radio frequency $(\mathrm{RF})$ field when placed in a strong magnetic field. Because magnetic resonance depends on each atom's local environment, chemists have long used NMR for clues to figure out the molecular structure of organic chemicals. For example, the ethanol molecule $\left(\mathrm{CH}_{3} \mathrm{CH}_{2} \mathrm{OH}\right)$ has three kinds of hydrogens: the three hydrogens attached to the terminal carbon, the two hydrogens attached to the middle carbon, and the one hydrogen attached to the terminal oxygen. In an NMR machine, the complex resonance pattern for each set of hydrogens appears in a slightly different part of the RF spectrum. The pattern

\footnotetext{
${ }^{4}$ Berger, "Magnetic Resonance Imaging" (2002).
} 
also reveals that the groups contain three, two, and one hydrogen atoms, respectively.

MRI applies this principle to a section of the human body: the body is placed into a large magnetic field, and then electricity pulsed through coils both to make systematic changes to the magnetic field and generate radio waves, systematically scanning through the threedimensional space. This is why MRI machines are so loud - the pulses also cause the coils to vibrate with significant force.

The atomic clock, NMR, and MRI all measure quantum effects. However, the technique that they use for detecting that effect is resonance with a radio frequency signal, which is based entirely on classical electronics.

Another first-generation quantum sensing technique is Positron Emission Tomography (PET), which uses small amounts of radioactive material to image metabolic processes in the body. ${ }^{5}$ Two-photon microscopy uses lasers to fluoresce tissues ${ }^{6}$ including those in live animals. ${ }^{7}$ Many nations use gamma-ray and neutron detecting devices, known as radiation portal monitor systems, at their borders to detect attempts to smuggle radiological materials or radioactive weapons. The devices can also detect radioactive waste in hospitals and landfills. $^{8}$

Such passive detectors are commercially available from firms such as Bertin Technologies SAS.

Recent developments in the mastery of other quantum effects enable new advancements in quantum sensing. The next section turns to these approaches.

${ }^{5}$ M. A. Taylor and Bowen, "Quantum Metrology and Its Application in Biology" (2016).

${ }^{6}$ Svoboda and Yasuda, "Principles of Two-Photon Excitation Microscopy and Its Applications to Neuroscience" (2006).

${ }^{7}$ Holtmaat et al., "Long-Term, High-Resolution Imaging in The Mouse Neocortex through a Chronic Cranial Window" (2009).

${ }^{8}$ In December 1983, the cobalt-60 source from a radiation therapy device broke open on the way to a junkyard in Juarez, Mexico, just across the Rio Grande from El Paso, Texas. The capsule contained 6010 tiny silvery pellets which contaminated the bed of the truck. The truck was scrapped and its steel recycled, which contaminated 5000 metric tons of steel. This steel was used in appliances and construction materials in Mexico, the US, and Canada. Ultimately 109 houses had to be condemned and several people were exposed to radiation as high as 200 rads, a life-threatening amount. See Blakeslee, "Nuclear Spill at Juarez Looms As One of The Worst" (1984). A similar incident happened in Taiwan in 1982. See Hwang, J. B. Chang, and W. P. Chang, "Spread of 60Co Contaminated Steel and Its Legal Consequences in Taiwan" (2001). 


\subsection{Modern Quantum Sensing Approaches}

"Second-generation" quantum sensing technologies advance on the first generation because they directly use quantum effects, such as entanglement, superposition, spins of subatomic particles, or superconductivity. An example would be a device that illuminates a remote object with one of two entangled photons, and then inspects the retained photon to make a measurement that would not have been otherwise possible.

The challenge in quantum sensing is to create a system that can be sufficiently controlled and monitored so that the changes to the system's quantum state are the result of the target object and not intrinsic noise from the device itself. External noise causes decoherence in the sensor, shortening the time in which the sensing can take place. In some cases, sensing requires electromagnetic shielding and cryogenic cooling, adding expense and limiting the environments in which quantum sensors can be used. Because coherence times are short and sensors in a superposition ultimately report a binary outcome, second-generation quantum sensors typically require many repeated measurements. Some approaches use ensembles of sensors so that these measurements can be performed in parallel.

Scientists are experimenting with more than a dozen kinds of quantum sensors that attempt to measure magnetic fields, electric fields, gravity, temperature, pressure, rotation, acceleration, frequency and time. ${ }^{9}$ Key approaches include:

\section{Superconducting Quantum Interference Devices (SQUIDs)}

These are magnetometers based on "Josephson junctions," circuits that sandwich a small insulating material between superconducting loops. ${ }^{10}$ The SQUID is connected to a detection coil, the shape of which can be matched to sensing needs. SQUIDs can detect the strength and gradient of magnetic fields, and since electrical current creates a magnetic field, SQUIDs can be used for non-invasive imaging of the human body. ${ }^{11}$ In the medical field, SQUIDs have been

\footnotetext{
${ }^{9}$ Degen, Reinhard, and Cappellaro, "Quantum Sensing” (2017).

${ }^{10}$ Josephson junctions are named after their inventor, Brian David Josephson, who won the 1973 Nobel Prize "for his theoretical predictions of the properties of a supercurrent through a tunnel barrier, in particular those phenomena which are generally known as the Josephson effects." Josephson predicted the effect as a 22-year-old $\mathrm{PhD}$ candidate at the University of Cambridge.

${ }^{11}$ Fagaly, "SQUID Magnetometers" (2014).
} 
used for analysis of the heart (magnetocardiography), the lungs, and to record brain activity (magnetoencephalography). ${ }^{12}$ SQUIDs also have myriads of uses outside healthcare. For example, SQUIDs can be used for detecting corrosion rates as small as 70-millionths of an inch per year in aluminum, ${ }^{13}$ as well as performing other kinds of non-destructive evaluation of materials. ${ }^{14}$

Among the oldest and most sensitive quantum sensors, SQUIDs are commercially available from firms such as US-based Quantum Design Incorporated and UK-based Cryogenic Limited. The SQUID sensor and coil are enclosed in a supercooled, vacuum-insulated container, so they are physically separated from sensed objects and, as such, currently cannot be used in living subjects. Superconducting circuits are the basis for many companies' quantum computing efforts, including Google, IBM, Intel, BBN (Raytheon), and Rigetti. ${ }^{15}$

SQUIDs may eventually be replaced by Optically Pumped Magnetometers (OPMs), devices that do not require cryogenic cooling. ${ }^{16}$ Today individual SQUID sensors can be purchased for a few thousand dollars, while fully functioning SQUID magnetometers easily cost hundreds of thousands of dollars.

Atomic vapor technologies These sense electric and magnetic fields with atoms suspended in a resonant medium. Atomic vapor technologies are promising because they can be initialized and read optically, and they operate at room temperature. Two promising variants of this technology are Electromagnetically Induced Transparency (EIT) and Spin Exchange Relaxation Free (SERF) magnetometry. In EIT, an otherwise opaque medium exhibits transparency when two lasers of different frequencies are pumped into it. Measurement of the transparency can detect subtle magnetic fields. ${ }^{17} \mathrm{In}$

${ }^{12}$ Heidari and Nabaei, "SQUID Sensors" (2019).

${ }^{13}$ Juzeliunas, Y. P. Ma, and Wikswo, "Remote Sensing of Aluminum Alloy Corrosion by SQUID Magnetometry" (2004).

${ }^{14}$ Faley et al., "Superconducting Quantum Interferometers for Nondestructive Evaluation" (2017); Jenks, Sadeghi, and Wilkswo Jr., "Review Article: SQUIDs for Nondestructive Evaluation" (1997).

${ }^{15}$ Buchner et al., "Tutorial: Basic Principles, Limits of Detection, and Pitfalls of Highly Sensitive SQUID Magnetometry for Nanomagnetism and Spintronics" (2018).

${ }^{16}$ Tierney et al., "Optically Pumped Magnetometers: From Quantum Origins to Multi-Channel Magnetoencephalography" (2019).

${ }^{17} \mathrm{EIT}$ is exciting because it can produce what is known as a "slow light" effect, when optical pulses travel through a medium with a low group velocity. This 
SERF, a high-density vapor of alkali atoms is polarized with a laser to an initial state. After being exposed to the magnetic field, a second probe light is used to detect changes in the atoms' polarization from the magnetic field. ${ }^{18}$

Despite active research on these physical phenomena, there is no evidence of an emerging commercial marketplace for atomic vapor technologies.

Nitrogen vacancy These approaches exploit imperfections in diamond crystals, that is, where a single nitrogen atom is trapped by the strong bonds of neighboring carbon atoms, and remains relatively insulated from the outside world. A laser is used to initialize the state of the nitrogen atom, and based on photons emitted from the crystal, one can measure magnetic fields at room temperature. Although artists sometimes illustrate articles on nitrogen vacancy with images of large diamonds, the size found on the ring fingers of the rich, in reality the "diamonds" are nanoscale thin membranes. Nitrogen vacancy diamonds are synthetic diamonds made by chemical vapor deposition (CVD), a process involving irradiation and annealing. ${ }^{19}$

Nitrogen vacancy devices are small enough to measure magnetic fields in vivo. ${ }^{20}$ To speed measurement, they can be arranged in an ensemble, but controlling these ensembles remains a key technical challenge.

Nitrogen vacancy is entering the commercial market. Swiss-based QZabre LLC offering a microscope integrating the approach, and precursor materials, such as CVD diamond films sold by Delawarebased Applied Diamond Inc. Nitrogen vacancy is also considered a promising medium for quantum computing because it operates at room temperature, and it is being pursued by Australia-based Quantum Brilliance, Japan's Nippon Telegraph and Telephone Corporation (NTT), and research groups at Tu-Delft's QuTech, MIT Lincoln Labs, and at Oxford University.

effect makes EIT a candidate for quantum memory and for optical transistors. (L. Ma et al., "EIT Quantum Memory with Cs Atomic Vapor for Quantum Communication" (2015))

${ }^{18}$ Budker and Romalis, "Optical Magnetometry" (2007).

${ }^{19}$ Ruf et al., "Optically Coherent Nitrogen-Vacancy Centers in Micrometer-Thin Etched Diamond Membranes" (2019).

${ }^{20}$ Fujiwara et al., "Real-Time Nanodiamond Thermometry Probing in Vivo Thermogenic Responses" (2020). 
Photonic approaches These approaches to quantum sensing have several advantages over other approaches owing to the relative resilience of photons. Photons can be sent out into free space and still retain their critically important quantum phenomena - their "spin" (see Appendix B.3). Photonic approaches sometimes require cooling, but often not the supercooling used to maintain quantum states in other media.

Photonic sensing uses techniques such as light squeezing, entanglement, single-photon detection, optical interferometry, and quantum "dots." ${ }^{21}$ Light squeezing involves limiting the uncertainty of a light wave for some portion of its phase (and thus increasing the uncertainty in other portions of its phase) in order to reduce errors. Photonic entanglement approaches use a pair of photons which have been correlated in some specific way. The pair of photons are split and go in different paths. One of the photons is aimed at something, either to detect it or to illuminate it. The other photon is simply measured directly. In quantum illumination, entanglement enables one to discern reflected light from noise, making it possible to filter and produce a cleaner image. In theory, this would be useful for sensing objects with extraordinarily low reflectivity, such as aircraft designed to have minimal radar cross-sections - sometimes called "stealth" aircraft. ${ }^{22}$ These techniques require development of devices that can emit and detect single photons (see Figure 2.3).

In optical interferometry, a beam of light is split and then superimposed upon itself. ${ }^{23}$ A detector compares the phases of the superimposed beams, and the patterns reveal evidence of other phenomena. For instance, the Laser Interferometer Gravitational-Wave Observatory (LIGO) uses optical interferometry to detect minute changes in the fabric of space-time that result from the passage of gravity waves. ${ }^{24}$

${ }^{21}$ Pirandola et al., "Advances in Photonic Quantum Sensing" (2018); Flamini, Spagnolo, and Sciarrino, "Photonic Quantum Information Processing: a Review" (2018).

${ }^{22}$ Guha and Erkmen, "Gaussian-State Quantum-Illumination Receivers for Target Detection" (2009).

${ }^{23}$ We discuss in Section B.1.4 (p. 493) how Michelson and Morley famously used an interferometer in 1887 to show that there is no aether.

${ }^{24}$ The 2017 Nobel Price was awarded to Rainer Weiss, Barry C. Barish, and Kip S. Thorne "for decisive contributions to the LIGO detector and the observation of gravitational waves." See Abbott et al., "Observation of Gravitational Waves From a Binary Black Hole Merger" (2016). 
A range of sensing applications are being explored for quantum dots, including chemical detection (for instance, the presences of extremely small amounts of heavy metals, pollutants, and pesticides) and for biological purposes, such as monitoring drugs and DNA. ${ }^{25}$ Quantum dots are crystals fabricated so that they control the movement of electrons; the restriction of movement enables quantized emission of energy. Quantum dots can even be grown to absorb or emit certain wavelengths of light. In effect, quantum dots act as large, artificial atoms. Grown in lattices, quantum dots range from 10 nanometers to a single micrometer.

Like other sensing substrates, quantum dots are a candidate for building quantum computers. Such computers would use the spin of the outermost electron as the qubit. ${ }^{26}$ Quantum dots are also being considered for improving existing systems such as solar panels, and for quantum communications, as they can be tuned to emit single photons.

Lasers have been used in many scientific contexts for decades, and quantum photonics has reached significant commercial maturity as a result. Today hundreds of vendors sell various kinds of photon-based components and whole systems. One can readily find sellers of single-photon detectors, single-photon emitters, bucket and Charge Coupled Device (CCD) photonic detectors, beta barium borate crystals to generate entangled photons, lenses to manipulate light polarity, and of course lasers of all varieties. New Jersey-based ThorLabs even sells demonstration kits for colleges that illustrate how polarization in 3D movies works and a small tabletop Michelson Interferometer for just a few thousand dollars. One cannot buy a fully assembled photonic sensor for the advanced applications discussed in this chapter, such as ghost imaging (see Section 2.3.4, p. 68), but one could purchase commercially the necessary components and assemble a ghost-imaging rig in a garage if one was so inclined (and had sufficient financial resources).

Several research groups are pursuing photonics as a medium for quantum computing, including Paris-based Alice\&Bob, the UK's Orca Computing, Swiss-based ID Quantqiue, California's PsiQuantum, and Canada-based Xanadu.

${ }^{25} \mathrm{M}$. Li et al., "Review of Carbon and Graphene Quantum Dots for Sensing" (2019).

${ }^{26}$ Loss and DiVincenzo, "Quantum Computation with Quantum Dots" (1998). 


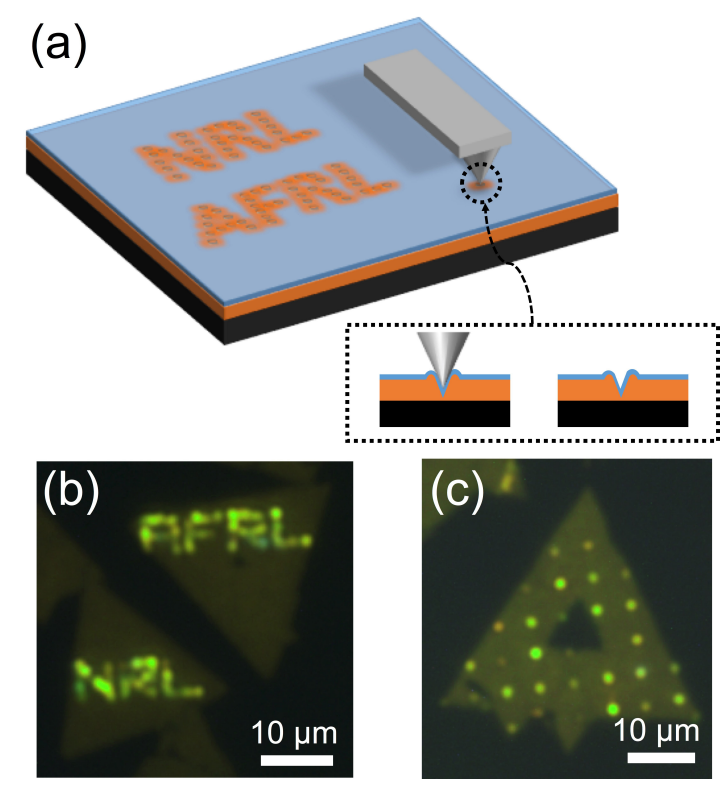

Figure 2.3. Scientists at the Navy and Air Force Research Laboratories placed 1-2 micrometer-sized light sources on semiconductors that can emit single photons on demand. Single-photon emitters and detectors are among the technologies that lower entry barriers for innovation in quantum technologies. Photo by Daniel Parry, courtesy of the US Naval Research Laboratory.

As quantum sensing improves, the world's measurement standards are getting upgraded as well.

Consider Le Grand K, the century-old piece of platinum iridium alloy in a secure underground vault in Paris. This metallic cylinder is 90 percent platinum by weight; in those 900 grams of platinum there are roughly $2.78 \times 10^{24}$ individual atoms. ${ }^{27}$ At least, that was the cylinder's weight when it was manufactured; measurements made in 1988 found that Le Grand K had lost roughly five-hundredths of a milligram - perhaps the result of improper handling, or perhaps the result of the material somehow outgassing. ${ }^{28}$

The problem with the cylinder's weight change mentioned in the preceding paragraph is that it is logically inconsistent. In $1988 \mathrm{Le}$ Grand $\mathrm{K}$ was the world's reference standard. If Le Grand $\mathrm{K}$ had

${ }^{27}$ Platinum has an atomic mass of $195.078 \mathrm{amu}$ (atomic mass units); to convert kilograms to amu, divide by $9.223 \times 10^{18}$. For a discussion of quantum sizes, see Appendix A.

${ }^{28}$ Keats, "The Search for a More Perfect Kilogram" (2011). 


\section{-The Origins of The Metric System}

Article 1, Section 8 of the US Constitution grants Congress the power "To coin Money, regulate the Value thereof, and of foreign Coin, and fix the Standard of Weights and Measures." That is, Congress has the power to determine how things in the US are weighed, how lengths are measured, how time will be kept, and so on. Other world governments claim similar powers. Fair and consistent taxation required a single system for money and a consistent set of measurements, given that many taxes were tariffs collected on a measure of a thing being imported.

By 1787 there was also wide realization within the scientific community that the exchange of scientific knowledge also required a consistent set of weights and measures, and efforts to create a standardized set of measures had been underway for some time. In 1790, the French Academy of Sciences was charged by the National Assembly to devise a new system of weights and measures. Over the next five years the Academy devised the Metric System.

The French Academy originally defined the meter as one 10-millionth the distance at the surface of the Earth from the North Pole through Paris to the equator; this is why the diameter of the Earth is $6371 \mathrm{~km}$ : the circumference was defined to be $10000000 \mathrm{~m}(10000 \mathrm{~km})$ and circumference $=2 \times \pi$, so $40000 \div(2 \times \pi)=6366$. The survey was completed in 1798 , at which point a bar of platinum was created to be the primary reference standard meter from which all others would be measured. (Platinum was used so that the length would not be affected by oxidation.) In 1959 the foot was redefined by the international yard and pound agreement to be exactly 0.3048 meters.

The kilogram was defined based on the meter and pure water: it is the mass of 1 liter of water (a liter is the volume of a cube that is $10 \mathrm{~cm}$ on a side). But as with the meter, this formal definition gave way to a platinum reference standard, the Kilogramme des Archives, which was cast in 1799. 
somehow lost a fraction of the atoms that made up its mass, in principle all of the world's scales should have required recalibration to the cylinder's new mass. That didn't happen because even though officially Le Grand $\mathrm{K}$ was the reference standard, in practice scientists had created near-perfect replicas of the standard, used them to develop extraordinarily accurate scales, and then used the consensus of all of those physical objects to reason about Le Grand K's changed composition. It is as if the scale at Le Grand K's gym, its doctor's office, and its best friend's house all said that it had lost a little weight, even though K's bathroom scale said that it weighed the same as it did back in 1889. Who are you going to believe?

The situation is even worse: as scales became increasingly accurate in the twentieth century, they became increasingly able to detect minor variations in the weight of nearly all of the kilogram's official copies. ${ }^{29}$ In part this is because the metallic surfaces absorb or release molecular impurities in the air - and even the air itself. It may also be a result of the wear that results from the need to physically handle these objects in order to measure them. So over the past fifty years, metrologists have worked diligently to redefine all of the standards of measurement in terms of quantum processes or measurements, just as the second was redefined in 1967 (see the sidebar "Defining and Redefining the Second" on page 35).

From a public policy perspective, moving the world's scientific standards from the measurement of specific physical objects to measurements of plentiful and identical quantum objects is democratizing. The movement means that any government, organization or individual with sufficient technical capabilities can make measurements as repeatable, as precise, and as accurate as they are able, without stopping to calibrate their measuring devices against some reference standard in Paris, France or Gaithersburg, Maryland. Previously, those groups could make measurements that were repeatable and precise, but accuracy depended upon performing that repeatable and precise measurement on a national standard. Thus it was a great step forward not just for science, but for the practice of science, when in 1960 the meter was redefined to be the length equal to 1650763.73 wavelengths of radiation in vacuum for a specific transition of krypton- 86 .

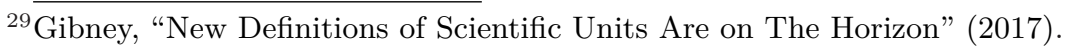


Surprisingly, the kilogram itself wasn't redefined until 2019 as part of the 2019 redefinition of the International System of Units (abbreviated SI from the French-language name Système International d'Unités). ${ }^{30}$ According to the English version of the standard:

the kilogram will continue to be the unit of mass, but its magnitude will be set by fixing the numerical value of the Planck constant to be equal to exactly $6.62606 \mathrm{X} \times 10^{-34}$ when it is expressed in the SI unit $\mathrm{m}^{2} \mathrm{~kg} \mathrm{~s}^{-1}$, which is equal to $\mathrm{J}$ s.

(The symbol X represents additional digits that were added in a technical memorandum. The SI value of Planck's constant is currently $6.62607015 \times 10^{-34}$.)

As in other areas of quantum information science, the improved metrology creates both the tools and the economic incentives to further improve metrology: this is another example of a virtuous circle.

\subsection{Quantum Sensing Applications}

At first it may seem to a non-scientist that there are few compelling commercial needs to be able to measure objects to within a nanometer (one-billionth of a meter) or time events to the nearest picosecond (one-trillionth of a second). After all, yardsticks and stopwatches seem like they are good enough for most day-to-day measurements. While it is true that the original motivation for making precise measurements was that of scientists seeking to have a better understanding of the natural world, many of the foundations of our modern technological society depend upon the ability to make measurements that are precise, repeatable, and accurate according to established international standards.

The wide range of quantum sensing technologies currently under development promise a new generation of measurement technology that is not only more precise and accurate, but also widely available and (eventually) low-cost. Critical to all these applications is more precise measurement of time and location, which is discussed next.

\subsubsection{Measuring Time}

Measuring time with more absolute accuracy and higher precision are the first benefits of quantum sensing; they are also requirements for

${ }^{30} \overline{\text { Bureau International des Poids }}$ et Mesures, The International System of Units (n.d.). 
breakthroughs in sensing other kinds of modalities. This is because precise measurement of time contributes to increases in precision for all other forms of sensing.

The Global Positioning System (GPS) is an example of the power that precise time measurement confers. Mathematically, the accuracy of GPS comes from the stability of orbital mechanics, Einstein's equations of relativity, and precise timekeeping. Each GPS satellite is individually numbered and orbits the Earth along a predetermined path. The satellite sends a radio signal down to the planet below consisting of the satellite's number, coefficients for various mathematical equations that allow computing the satellite's position at any given time, and the precise time that the radio wave left the satellite's antenna, as defined by the satellite's on-board atomic clocks.

GPS receivers listen for these signals from the satellites. If a receiver can "hear" and resolve signals from three satellites, it can solve a series of equations and determine its latitude, longitude, and the precise time. This is possible because all of the satellite clocks are synchronized, but because the distance between the receiver and each satellite is different, the timestamp on each received signal is slightly divergent. Thus, the distance to a specific satellite is simply the receiver's computed time minus the time that a specific satellite is reporting.

Light moves at $299792458 \mathrm{~m} \mathrm{~s}^{-1}$ - roughly $30 \mathrm{~cm}$ every nanosecond. This means that if the clock on the satellite were to lose or gain just 10 nanoseconds, the satellite's computed position would be off by 3 meters. In practice, such precision in the rigors of outer space requires more than just an atomic clock: it also requires compensating for the impact of time dilation caused by each satellite's orbital speed of roughly $3.9 \mathrm{~km} \mathrm{~s}^{-1},{ }^{31}$ which causes the satellite's atomic clocks to tick slightly slower than they would on Earth. The GPS receiver computes your speed from the Doppler Shift of each incoming radio signal: if it is at a slightly higher frequency than expected, then the distance between you and the satellite is decreasing; if it is lower than expected, the distance is increasing. In practice, GPS receivers are so sensitive that they are able to measure the speed of a person walking from its impact on the Doppler Shift.

GPS clocks are pretty accurate, but they do drift. Twice each day, each satellite synchronizes its internal atomic clocks with a ground

${ }^{31}$ Caro et al., "GPS Space Segment" (2011). 


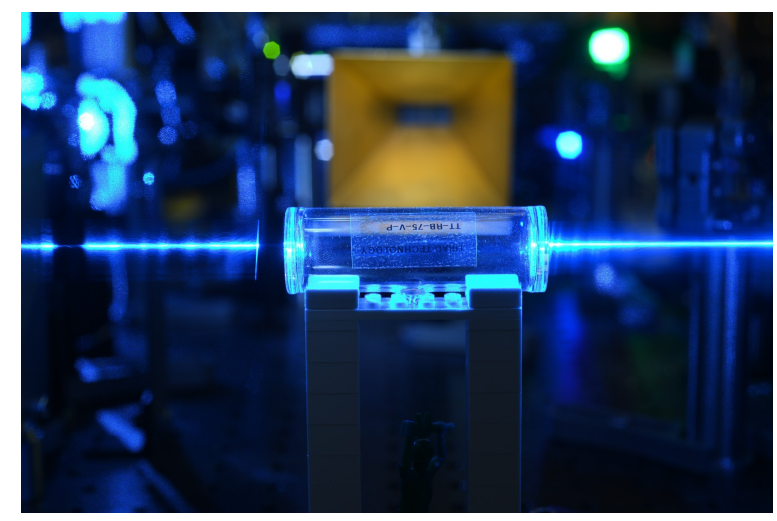

Figure 2.4. US Army Research Laboratory researchers tested Rydberg atoms' sensitivity, finding them to be sensitive to radio waves across the entire spectrum. Rydberg atom sensors may enable new ways to detect stealthy communication and without the inconvenience of multiple antennae, because the laser that excites the atoms can tune the sensor to detect desired frequencies. Image courtesy of US Army.

station. NASA has also developed deep space atomic clocks based on ion traps and mercury ions for applications where the clocks cannot be readily updated. ${ }^{32}$

Another application for precise timing is to increase the resolution of individual sensors by making repeated exposures and combining them. This approach is sometimes called "super resolution." Photographers can do it today by taking four or eight photos of the same scene and then combining the images using software: this technique requires having the same exposure with each photo. But the same approach can be applied in principle to all kinds of measurements.

Metrologists are now developing approaches for creating so-called optical clocks that measure vibrations of atoms in the optical region of the radio spectrum, where light cycles roughly a million times faster than it does in the microwave region used by today's atomic clocks. Using ion trap technology the National Institute of Standards and Technology (NIST) built optical clocks based on the vibration of a single mercury ion (in 2006) and a single aluminum ion (in 2010).

Highly accurate clocks can measure subtle changes in gravity, thanks to the way that the flow of time changes depending on the clocks' position in a gravity well, as predicted by Einstein's general

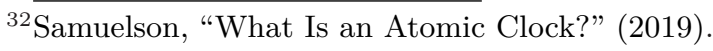




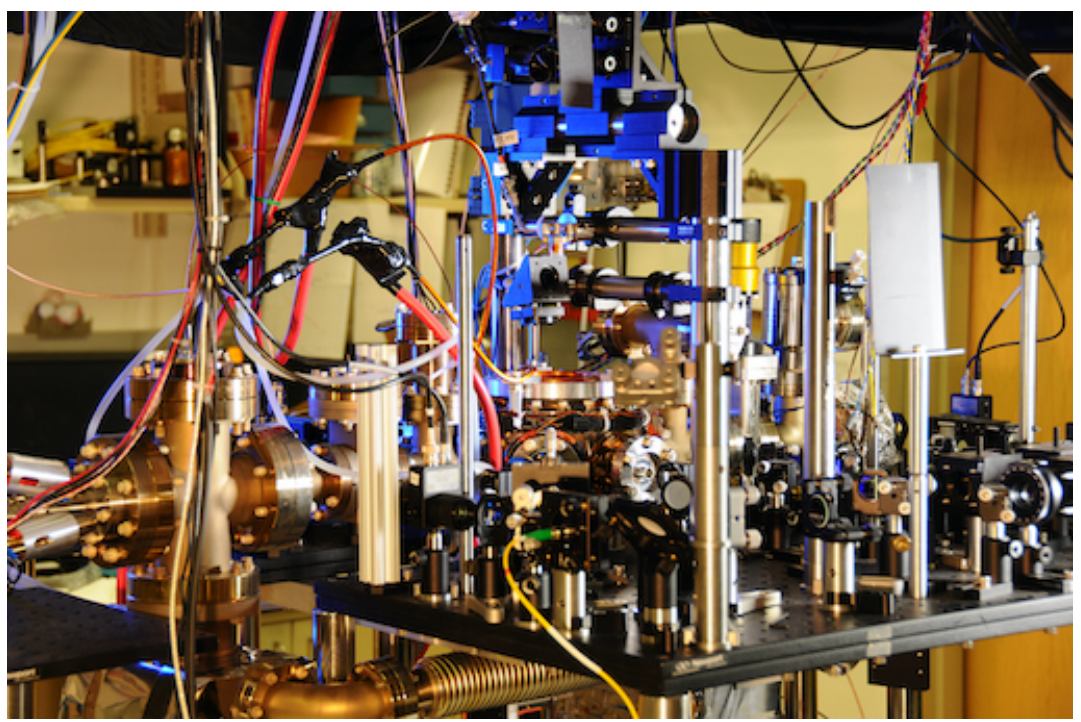

Figure 2.5. The National Institute of Standards and Technology developed this atomic clock based on an ytterbium lattice in 2013. Image public domain by Burrus/NIST.

theory of relativity. As NIST scientists explained, "if two identical clocks are separated vertically by $1 \mathrm{~km}$ near the surface of Earth, the higher clock emits about three more second-ticks than the lower one in a million years." 33 In that same 2010 paper, NIST reported that its atomic clock was sensitive enough to detect an up-or-down movement of just $33 \mathrm{~cm}$.

In 2018 NIST announced a breakthrough for their atomic clock based on a lattice of ytterbium atoms (going back to the mid-1950s, previous clocks were based on cesium). ${ }^{34}$ This clock will contribute to geodesy, the study of the shape, orientation in space and gravitational field of the Earth. The ytterbium atomic clock can make geodesic observations to within a centimeter accuracy. ${ }^{35}$ This clock, and another clock based on strontium ions, are so accurate that one of these clocks would neither gain nor lose a second if it were left running for more than 10 billion years (assuming, of course, that

${ }^{33}$ Chou et al., "Optical Clocks and Relativity" (2010).

${ }^{34}$ McGrew et al., "Atomic Clock Performance Enabling Geodesy Below The Centimetre Level" (2018).

${ }^{35}$ Gaithersburg, MD: National Institute of Standards and Technology, "Second: The Future" (2019). 
the rest of the clock could be engineered with the required level of reliability). ${ }^{36}$

The next stage goal is to move beyond measuring the oscillation of electrons as is performed in today's atomic clocks to the "nuclear clock," that measures time by focusing on the states of an atomic nucleus.

The Defense Advanced Research Projects Agency (DARPA) and private-sector investments in quantum sensing have resulted in more accurate atomic clocks, ${ }^{37}$ and smaller devices that are now commercially available. For instance, Microsemi Corporation sells a "chip scale atomic clock" that is only 35 grams. "Today's microwave-based atomic clocks on GPS satellites provide 10-nanosecond (billionth of a second) timing, whereas optical clocks could provide 10-picosecond (trillionth of a second) precision," explains a DARPA brochure on the quantum technology projects section of the agency's website. ${ }^{38}$

\subsubsection{Sensing Location}

Since the 1990s the primary source of positioning (determining where one is and orientation), navigation (determining one's desired position and routes to it), and timing (determining accurate and precise time) information are complex systems built from satellites that orbit the planet, ground stations that service those satellites, and billions of handheld receivers that sense the extraordinarily faint radio signals from the satellites and use them for the basis of complex mathematical operations. Although these systems are typically called GPS, after the US Global Positioning System, there are actually four competing positioning, navigation, and timing (PNT) systems in the world today.

Satellite navigation has become such a critical part of both the modern economy and the modern military that the Russian Federation, Europe, and China have all spent billions of dollars developing and fielding their own systems (see Table 2.1), so that they will not be dependent upon the continued diplomatic goodwill of the United States.

${ }^{36}$ Chou et al., "Optical Clocks and Relativity" (2010).

${ }^{37}$ Nicholson et al., "Systematic Evaluation of an Atomic Clock at $2 \times 10^{-18}$ Total Uncertainty" (2015).

${ }^{38}$ Defense Advanced Research Projects Agency, "Quantum Sensing and Computing" (2020). 
Table 2.1. Satellite navigation systems

Year

\begin{tabular}{cll} 
operational & Sponsor & System \\
\hline 1990 & US & Global Positioning System (GPS) \\
1993 & Russia & $\begin{array}{l}\text { GLONASS } \\
\text { Global Navigation Satellite System } \\
\text { (GNSS or Galileo) } \\
\text { Beidou } \\
\text { (Běidǒu Wèixing Dǎoháng Xitǒng) }\end{array}$ \\
\hline
\end{tabular}

\section{GPS History}

The US Global Positioning System (GPS) satellites were the first satellite-based navigation system; GPS became operational in 1990, 12 years after the launch of the first GPS satellite in 1978. Although the system was designed and funded and intended for use by the US military, provisions were made for incidental use by civilians as well.

Each GPS satellite has a synchronized atomic clock and sends information about its location and current time that can be received anywhere on Earth's surface or in the air (see Section 2.3.1, p. 47). Although it is commonly believed that the satellites track the receivers, this is not the case. Just as a boat at sea determines its position from observing a lighthouse, each GPS satellite tracks itself, and each receiver determines its position by finding and tracking the satellites.

Each US GPS satellite broadcasts on multiple frequencies, including a set of civilian frequencies that are open for public use and one or more military frequencies that are protected by various technologies. In March 1990, shortly after the GPS system became available, the US government intentionally made the civilian signals less accurate through a system called selective availability, which increased the uncertainty of civilian receivers from $20 \mathrm{~m}$ to $100 \mathrm{~m} .{ }^{39}$ This was done so that foreign militaries and terrorist organizations could not use the high-resolution GPS signal against the interests of the US.

But shortly after selective availability was switched on, the US found itself at war in the Persian Gulf with Iraq. There was a shortage of military-grade GPS receivers, and civilian-grade GPS receivers

${ }^{39}$ Thorton, "Selective Availability: A Bad Memory for GPS Developers and Users" (2018). 
were sent from the US to warfighters in the theater. ${ }^{40}$ The US responded by disabling selective availability until the conclusion of the war in July 1991, when it was promptly turned back on.

Even with selective availability, GPS found increasing uses in the civilian economy. Although a handheld GPS receiver cost a few hundred dollars, GPS became wildly successful in the civilian marketplace. While the designers of GPS had expected that it would be used in boating, aviation and by hikers, in-car navigation systems soon appeared on the market, a result of the navigation revolution working synergistically with the computer revolution. Selective availability was a constant annoyance for these systems, so approaches were found to get around it.

Differential GPS (DGPS) was one approach for addressing the error introduced by selective availability. DGPS uses a second set of ground stations that "listened" to the GPS signal, figured out how much error was being introduced by selective availability at that very moment, and sent out a correction. Two proponents of DGPS in the US government were the Federal Aviation Administration and the US Coast Guard, both of which had stakeholders that required high-precision PNT to allow for instrument navigation at night and during inclement weather. The fact that two different parts of the US government couldn't agree on how GPS should be controlled, and that each was willing to spend money and engineering effort to deploy a system that advanced its interests in a manner that was antagonistic to another government agency, should be carefully noted.

As commercial use grew, so did pressure on the US government to permanently switch off selective availability. This finally happened on May 1st, 2000. The newest GPS "Block III" satellites, first launched in December 2018, do not even have selective availability capability. In part this may be because the multiplicity of satellite-based PNT makes a satellite-based system such as selective availability less relevant: if the US switched on selective availability, a US adversary could simply use the Russian system instead.

Today you can purchase a 72-channel satellite navigation receiver from China for just $\$ 4$; the package is just 34 by 28 by $9 \mathrm{~mm}$ and includes an embedded antenna (alas, battery not included). The chip

40"GPS Navigation: From The Gulf War to Civvy Street" (2018). 
works with both the US and the Russian systems, presumably allowing users to align their PNT supply chains with their geopolitics.

\section{GPS Spoofing and Jamming}

The signals from navigation satellites are quite weak by the time they reach the Earth, leaving all satellite navigation systems vulnerable to spoofing or jamming by stronger signals. Attacks on GPS are motivating investment in quantum PNT approaches.

GPS spoofing is the act of generating radio signals that confuse GPS receivers into thinking that they are in one place when they are really someplace else. In December 2011, the Christian Science Monitor reported that the Iranian military had stolen a US bat-wing RQ170 Sentinel unmanned aerial vehicle by spoofing the GPS signals that the drone received. ${ }^{41}$ Likewise, Russia may use GPS spoofing to mask the whereabouts of its high-ranking officials. ${ }^{42}$ The Russian armed forces are renowned for their electronic warfare prowess on the battlefield, ${ }^{43}$ meaning that local jamming may disrupt equipment in a specific conflict. Increasingly, there is anxiety that satellites themselves will be attacked, ${ }^{44}$ leading to a regional or global outage.

GPS jamming is a simpler attack in which the faint GPS signals are simply overrun by other signals on the same frequency. Today GPS jammers that plug into an automobile's cigarette lighter can be purchased for as little as $\$ 10.79$ from the Walmart website. ${ }^{45}$

${ }^{41}$ Peterson and Faramarzi, "Exclusive: Iran Hijacked US Drone, Says Iranian Engineer" (2011).

${ }^{42}$ C4ADS, "Above Us Only Stars: Exposing GPS Spoofing in Russia and Syria" (2019).

${ }^{43}$ Creery, "The Russian Edge in Electronic Warfare" (2019); McDermott, Russia's Electronic Warfare Capabilities to 2025 (2017).

${ }^{44}$ Kan, China's Anti-Satellite Weapon Test (2007).

${ }^{45}$ Such devices are popular with truckers, as they defeat GPS vehicle trackers, allowing the truckers to take unauthorized routes or drive over the speed limit without the rig's owner taking notice. In 2013 a man in New Jersey was fined $\$ 31875$ by the Federal Communications Commission for operating such a device near Newark Liberty International Airport, where it interfered with aircraft operations. See Strunsky, "N.J. Man Fined $\$ 32 \mathrm{~K}$ for Illegal GPS Device That Disrupted Newark Airport System" (2013). Indeed, notes the FCC, "The use of a phone jammer, GPS blocker, or other signal jamming device designed to intentionally block, jam, or interfere with authorized radio communications is a violation of federal law" and "It is also unlawful to advertise, sell, distribute, import, or otherwise market jamming devices to consumers in the United States." US Federal Communications Commission, "Jammer Enforcement" (2020). 
The US government uses the term "denial" to describe technologies such as spoofing and jamming that can deny the use of GPS to an adversary. Developing these technologies has been part of US strategy since selective availability was turned off. This is clearly signaled on the government's GPS website: "The United States has no intent to ever use SA again. To ensure that potential adversaries do not use GPS, the military is dedicated to the development and deployment of regional denial capabilities in lieu of global degradation." 46

Of course, other governments are developing similar technology. The US Navy is responding, in part, by training midshipmen and navigators to use charts and sextants so that they will have a low-tech navigational fallback (see Figure 2.6). ${ }^{47}$ Concerns about attacks on satellites were one of the factors behind the elevation of the US Space Command to the status of being a unified combatant command. ${ }^{48}$

\section{Inertial Navigation}

Inertial navigation is an alternative to both GPS and celestial navigation. These systems use a combination of on-board accelerometers and gyroscopes to continuously track changes in a vehicle's motion and orientation. This approach to navigation, called dead reckoning, is sort of like closing your eyes while you are walking down a sidewalk with the goal of walking another 50 feet and then stopping at the traffic light to press the "walk" button.

The first inertial navigation system (INS) was designed and built by Robert Goddard at his research facility in Roswell, New Mexico, with its first successful demonstration in September 1931. Goddard's work was largely ignored by the US Government but was replicated and extended by Wernher von Braun in Nazi Germany, who perfected the system and used it to guide Germany's V2 rockets to their targets. "More than 9000 civilians and soldiers were killed in total in V2 attacks on the Allies. That excludes the estimated 12000 labourers and concentration camp prisoners killed while making the

\footnotetext{
${ }_{46}$ National Coordination Office for Space-Based Positioning, Navigation, and Timing, "Frequently Asked Questions About Selective Availability" (2001).

${ }^{47}$ Brumfiel, "US Navy Brings Back Navigation by The Stars for Officers" (2016).

${ }^{48}$ The Space Force, originally formed and housed in the Air Force out of Vandenberg Air Force Base, now has the leadership of a four-star general and a seat at the Joint Chiefs of Staff. Space Command now has authority over all military actions in space, defined as $100 \mathrm{~km}$ above sea level.
} 


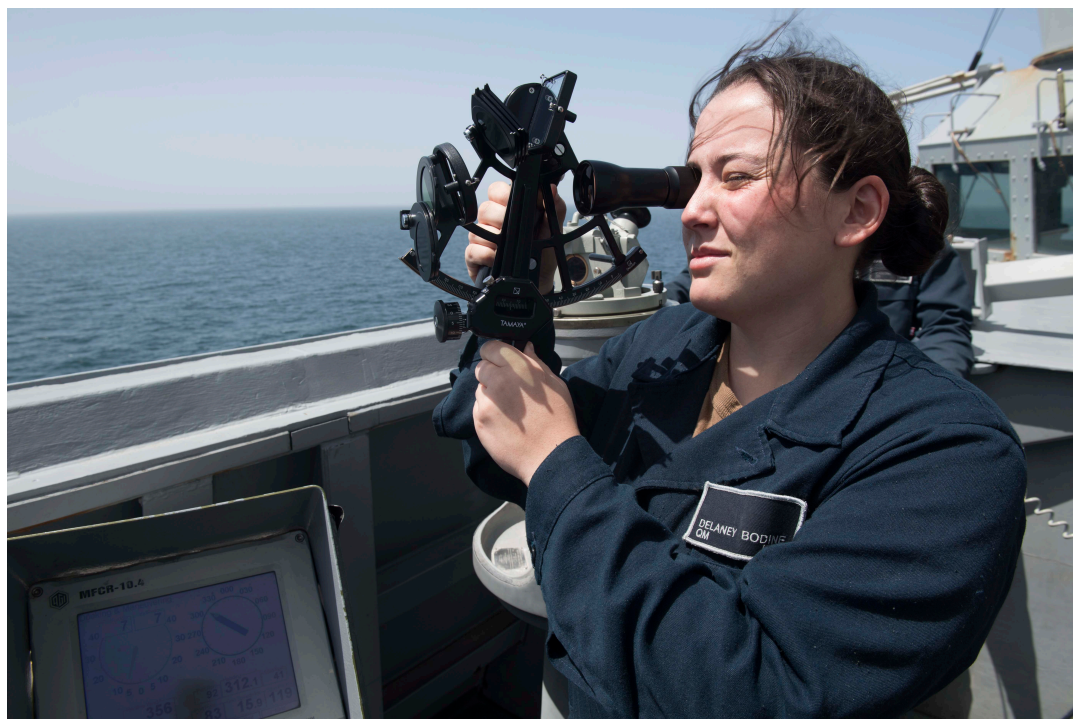

Figure 2.6. Quartermaster Seaman Delaney Bodine, from Elkton, Maryland, uses a sextant on the bridge wing while standing watch as the Boatswain's Mate of the Watch (BMOW) aboard the guided-missile destroyer USS Spruance (DDG 111) in the Arabian Gulf, March 20, 2019. In 2011 the Navy's Surface Warfare Officers School in Newport, RI, resumed training in celestial navigation for navigators and assistant navigators. Navy photo by Mass Communication Specialist 1st Class Ryan D. McLearnon/Released.

missiles." 49,50 After the war, von Braun was invited by the US government's Operation Paperclip to continue his research in the United States. A parallel effort to develop inertial guidance systems was initiated at the MIT Instrumentation Laboratory in the 1950s, which separated from MIT in 1973 to become The Charles Stark Draper Laboratory.

INS is another example of a dual use technology. Pan Am, once a great US airline, installed its first INS on a Boeing 707 in 1964. That early system had problems: because it was based on a gyroscope with mechanical bearings, it tended to drift over time. ${ }^{51}$ An improved

${ }^{49}$ Arkell, "Death From above without Warning: 70 Years after The First One Fell, Interactive Map Reveals Just Where Hitler's V2 Rockets Killed Thousands of British Civilians in Final Months of WW2" (2014).

${ }^{50}$ The BBC estimates that 20000 prisoners pulled from concentration camps died constructing the V2s. See Hollingham, "V2: The Nazi Rocket That Launched The Space Age" (2014).

${ }^{51}$ Morser, "Inertial Navigation" (2020). 
inertial guidance system that used gas bearings was deployed in the Boeing 747. Even so, the Federal Aviation Administration required that three INS units be installed in each cockpit, so that the pilots could rely on two systems at all times, with the third standing by as a hot spare. (The 747 was also equipped with a sextant port, as was the Vickers VC10, a British long-range jetliner produced in the 1960s.)

Today's mobile phones can also perform inertial guidance, thanks to their MEMS (micro-electromechanical system) accelerometers and gyroscopes. Although the sensors are not accurate enough for extended dead reckoning, they provide sufficient accuracy that a person's precise location can be determined by fitting the patterns of acceleration, movement, and deceleration to a street map. ${ }^{52}$ This demonstrates one of the ways that external information can be used to increase the effective sensitivity of measuring instruments.

Quantum sensors offer the promise of dramatically improved inertial navigation. Just as the lower friction of gas bearings made gyroscopes less subject to drift than mechanical bearings, gyroscopes based on ring lasers and eventually cold atoms promise even more improvements.

As the name implies, ring laser gyroscopes are gyroscopes based on the principle of sending laser light around a ring: the light remains on its current path even if the ring moves while the photons are in flight, allowing the movement of the ring to be precisely measured. In 2017 the ring laser market was estimated to be at $\$ 720$ million, with a projected annual growth rate of 3.5 percent. "Ring laser gyroscopes are primarily implemented in defense applications owing to their excellent measurement accuracy and [the absence] of moving parts that are in mechanical gyroscopes." 53 Today a single ring laser inertial navigation system can be purchased from China for between one thousand and ten thousand dollars.

Dramatically more accurate gyrometers based on "cold atom" technology are now being developed. "For inertial navigation, atom interferometers are particularly important because they provide an absolute measurement of the physical quantity of interest, be it acceleration or rotation...In geophysics, a gyrometer can be used for local

${ }^{52}$ Jun Han et al., "ACComplice: Location Inference Using Accelerometers on Smartphones" (2012).

${ }^{53}$ Research, "Ring Laser Gyroscope Market - Snapshot" (2017). 
monitoring of the variations in Earth's rotation rate due to seismic or tectonic-plate displacements." 54

The researchers developing inertial guidance for mobile phones matched acceleration patterns against street maps of Pittsburgh and Mountain View to determine a mobile phone's location. ${ }^{55}$ Similarly, an advanced INS could in principle match changes in the Earth's magnetic field against a map to dramatically improve its accuracy. Such systems would work equally well underground or underwater. Today's submarines navigate using a variety of strategies, including GPS antennae attached to a long tether that can be sent to the surface and then either reeled back down or cut as necessary.

One could imagine vessels of all types being equipped with GPS, inertial guidance, and quantum magnetometers. Properly equipped, comparisons between GPS and the quantum sensor should reveal when GPS is being jammed or degraded, and tell the operator where the vehicle is located with certainty. An unclassified summary of a 2015 Air Force quantum technologies study concluded that quantum navigation sensors would be ready for demonstration between 2020 and 2025. ${ }^{56}$ Efforts are underway to miniaturize the devices so they can be used in all kinds of applications. For example, in 2019, MIT scientists created a microchip-sized nitrogen vacancy magnetometer using standard complementary meta-oxide-semiconductor (CMOS) technology, paving the way to small devices. ${ }^{57}$

\section{Quantum Sonar}

Because SQUID-based magnetometers and gravimeters can sense exceedingly minute changes in the Earth's magnetic and gravitational fields, these devices can be used to create three-dimensional models of the underground mineral deposits or man-made structures that are responsible for those changes. Such models are made by moving the sensor in three-dimensional space while precisely recording the location and orientation of the sensor, and then using a computer to fit a mathematical model of the presumed underground object

${ }^{54}$ Alzar, "Compact Chip-Scale Guided Cold Atom Gyrometers for Inertial Navigation: Enabling Technologies and Design Study" (2019).

${ }^{55}$ Jun Han et al., "ACComplice: Location Inference Using Accelerometers on Smartphones" (2012).

${ }^{56}$ US Air Force Scientific Advisory Board, Utility of Quantum Systems for The Air Force Study Abstract (2016).

${ }^{57} \mathrm{Kim}$ et al., "A CMOS-Integrated Quantum Sensor Based on Nitrogen-vacancy Centres" (2019). 


\section{Sensing and The Fundamental Forces}

There are four fundamental forces in nature: electromagnetism, gravity, and the strong and weak nuclear "forces." Of these, electromagnetism is the base of nearly all remote sensing today, as it is easy to generate, control, and measure. This ability to control also means that it is possible to shield from electromagnetic waves.

We currently lack the ability to generate, control, or shield from gravity waves. (Such an ability would presumably enable the anti-gravity and artificial gravity devices commonly seen in science fiction.) But we can detect gravity waves based on their interaction with other masses and, thus, with the fabric of spacetime. Increasingly precise quantum sensors create the opportunity for high-resolution gravity sensing. Today such techniques are creating new possibilities for both astronomy and geology, although increased resolution might create possibilities for even more precise measurements of human artifacts in the future.

The two remaining forces are mostly confined to the nucleus of the atom. The strong force, more properly called the strong interaction, is responsible for holding the atomic nucleus together: without it, the protons would repulse and matter as we know it would not exist. The weak interaction, meanwhile, is responsible for radioactive decay.

Electromagnetism and gravity can be used for remote sensing because they follow the inverse square law, which is to say, because the force that they exhibit between two objects is proportional to $\frac{1}{r^{2}}$ where $r$ is the distance between the two objects. The strong and weak forces do not follow the inverse square law. They are much stronger than the electromagnetism and gravity within and in the immediate vicinity of the atomic nucleus, but they appear to play no role at larger scales of measurement.

responsible for the disturbance in the magnetic force to the observations. In principle, such methods are no different from techniques that geologists have used for decades to explore for mineral wealth and oil. In practice, the exquisite sensitivity of SQUID-based sensors creates new opportunities for observing the hidden world.

It is likely that there will be many applications outside of the extractive industries for such underground sensing technology. For ex- 
ample, the Chinese military has reportedly developed next-generation, sonar-like systems that can detect submarines and other underground objects based on their mass and shape. ${ }^{58}$ Other publications describe how Chinese scientists flew a SQUID-based magnetometer over a field to detect buried iron balls of various sizes based on how the balls changed the Earth's magnetic fields. ${ }^{59,60}$ There are obvious applications for landmine detection.

The iron-ball-detecting device is fascinating. The scientists created a proof-of-concept by hand-carrying an array of six SQUIDs. Each SQUID in the device contained a 24-bit analog-to-digital converter (which means that its precision is $2^{-24}$ or one part in 16 million). Illustrating the importance of super-precise timing, the papers emphasized that the device could make 2000 measurements a second, with a time synchronization of 1 microsecond. The device included a sensitive inertial navigation system to know the precise location and orientation of the detector ensemble. By knowing the device's location and orientation, it is then possible to know the precise direction and magnitude of the Earth's magnetic field at a series of measurement locations - and remember, this device is making 2000 measurements each second.

The papers report that the scientists detected all of the iron balls. A follow-up experiment replicates the procedure, but the SQUID array is dangled from a helicopter with a towrope. Given the speed of measurement, the ability to know location, and orientation of magnetic fields, these devices should be able to detect the existence of underground tunnels or structures, and even the movement of military matériel or even drugs through such tunnels.

Many details of the helicopter experiment are vague. The experiment suggests that the helicopter approach worked, yet one paper says that the data were still being processed at time of publication. Other details, such as the altitude of the helicopter, whether it was modified to avoid interference with the device, the size of the balls, and so on, were either vague or omitted. Yet, a photograph in Qiu et al. gives some hints: it reports that the rope suspending the SQUID

${ }^{58}$ Hambling, "China's Quantum Submarine Detector Could Seal South China Sea" (2017).

${ }^{59} \mathrm{Wu}$ et al., "The Study of Several Key Parameters in The Design of Airborne Superconducting Full Tensor Magnetic Gradient Measurement System" (2016).

${ }^{60}$ Qiu et al., "Development of a Squid-Based Airborne Full Tensor Gradiometer for Geophysical Exploration" (2016). 
array is 35 meters long. Based on the length of the towrope, people's presence in the photograph, and its angle, the helicopter appears to be flying rather low, with the sensor perhaps 5-10 meters off the ground. The iron balls appear to vary in size from a golf-ball-sized object to one the size of a melon. But without a scale, it is hard to be more precise.

Such approaches for quantum sensing have obvious implications in counter-terrorism and counter-smuggling operations. But quantum sensing extended to the ocean might have implications for submarine tracking and anti-submarine warfare, which would have significant geopolitical repercussions for nuclear deterrence (see Figure 2.7). Since the 1960s, the US nuclear strategy has been based on the so-called nuclear triad and the serviceability of some US nuclear forces in the event of a massive first strike by another power. The most survivable of the nuclear forces are those in submarines, since the subs can remain underwater for months at a time and deliver a massive retaliatory second strike, with the intent of utterly destroying an attacking nation (and also ending all remaining human life on the planet in the process). Without survivable forces, game theory says that there is an incentive for a nuclear nation to strike first and wipe out its adversary's forces before they can get off the ground.

Quantum sensors that would allow an adversary to accurately pinpoint and track the location of an adversary's nuclear forces would appear to impact the survivalability of those weapons. If all such weapons can be tracked, it might be able to destroy them all in a single surprise attack. Thus, high-precision quantum sensing that could recognize mass distributions such as those that appear in Figure 2.8 might be destabilizing.

Aside from nuclear attack and counter-terrorism, there are certain hot zones where even a limited-range quantum sonar might change how countries posture. SQUIDs might improve the effectiveness or decrease the cost of minesweeping, for example, perhaps to the point of allowing for low-cost detection systems that could detect mines and even pirate vessels (significant research has already been devoted to landmine detection ${ }^{61}$ ). In areas such as the South China Sea, a magnetometer-based surveillance system might tip off a nation to the presence of another nation's underwater vessels and lead to their exclusion.

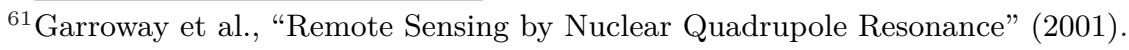




\section{Detecting the Gravitational Quadrupole of a Simple Submarine Model}

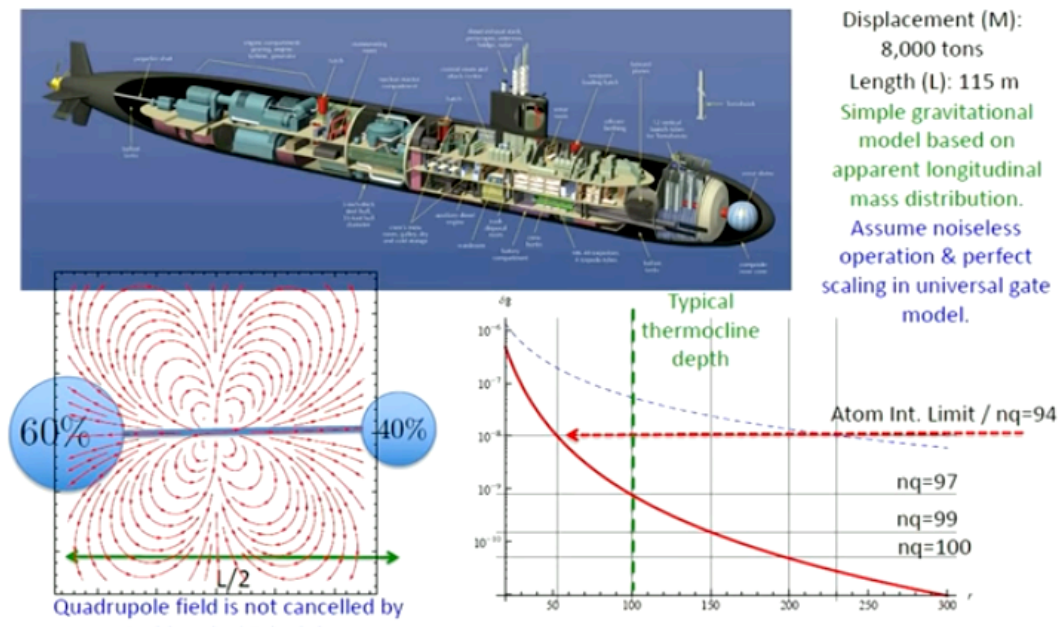

Figure 2.7. In a 2018 address to the National Academies, Dr. Marco Lanzagorta, explained how quantum gravimeters might detect a submarine. Image courtesy US Naval Research Laboratory.

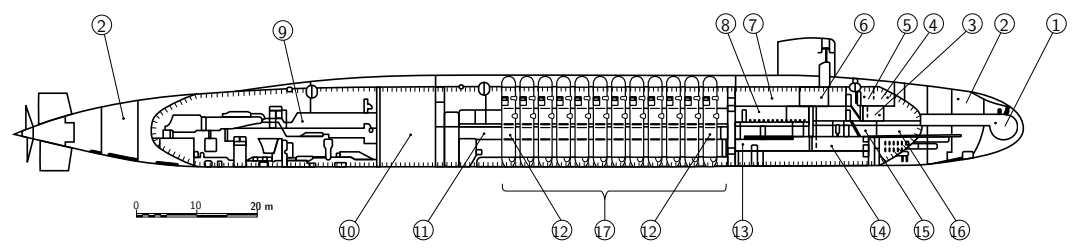

Figure 2.8. The engine room (9), reactor compartment (10), torpedo room (14), and missile compartment (17) in this Ohio class nuclear submarine provide several highly dense areas - arranged in a distinctive pattern unlikely to occur naturally in the ocean - that quantum sensing devices could detect. Image CC-By Wikimedia Commons user Voytek S. 


\section{Magnetic Field Sensing Futures}

Quantum sonar is an application of magnetic field sensing. As explained in Chapter 9, today it is militaries that are the principal patrons of quantum technology research, and perhaps that funding shapes our imagination for applications of quantum sensing. As quantum sensing matures, more entrepreneurs will understand the potential for the technology, and be able to actually deploy it. Sensing magnetic fields has enormous application that we sketch below. A wealth of applications could flow from the SQUID magnetometer approach, especially as the technology is miniaturized and becomes usable higher in the air or even in outer space.

- Extractive industries, ranging from oil and gas to technology companies desperate for the rare-earth minerals necessary for mobile phones, will benefit from sensing below-ground magnetic fields. A subsidiary owned by De Beers, in an effort similar to the Chinese iron-ball detecting experiment, suspended an array of six low-temperature SQUIDs 20 meters below a helicopter, and used the array to map the magnetic gradients over a $7 \mathrm{~km}$ square area in South Africa. ${ }^{62}$ The implications for extraction industries are clear. By mapping magnetic and gravimetric waves, these industries should be able to locate valuable minerals, allowing for exploration and mining that is dramatically more effective than before - and thus lowering the net price of extracted materials. The implications for the environment are less clear: will such technologies allow for highly targeted extraction, or more extraction overall?

- Scientists interested in brain-machine interfaces could use quantum sensing to detect subtle electrical signaling in the brain. Signaling in the brain is fainter than the electrical field created by the heart, requiring more sensitive instrumentation and advances in locating and isolating brain signals. It might also allow for improved lie detectors and even brain wiretapping. ${ }^{63}$

- Medical treatment and research centers, as evidenced by broad adoption of MRI and PET, were quantum sensing early adopters.

${ }^{62}$ Chwala et al., "Full Tensor SQUID Gradiometer for Airborne Exploration" (2012).

${ }^{63}$ Garfinkel, Database Nation: The Death of Privacy in The 21st Century (2000). 
As quantum sensors improve in resolution, there will be corresponding benefits to diagnosis and treatment.

- Today conventional magnetometers are widely used to detect weapons. In the future, machine learning might be used to allow the identification of popular rifles and handguns, in all their possible orientations. With such a model, a magnetometer might be able to detect all guns within a specific range while ignoring other items. Using an airborne magnetometer, firearms might be easily detected in a crowd, or even in homes and vehicles because of the lack of shielding. This might be used to find weapons that are unregistered or that are possessed by people ineligible to own firearms; courts would need to decide if flying a magnetometer over a neighborhood or crowd constituted a search that required a warrant.

- Quantum sensing might allow for dramatically smaller antennae in consumer and professional electronics (see Figure 2.4). For example, a paper from the Delft University of Technology in Delft, Netherlands, reports that a supercooled sensor was able to detect single quanta of radio waves. ${ }^{64}$ A 2020 paper by the Army Research Laboratory demonstrated that Rydberg atoms (atoms with excited valence electrons) were sensitive to the entire radio band spectrum. ${ }^{65}$ The future of radio communications may not rely on bigger antennae or stronger transmission but rather on more sensitive sensing and narrower allocations of frequency spectrum. This is in part because the lasers controlling the sensors can tune focus to specific frequencies without reliance on multiple, different antennae. More sensitive radio would augur more efficient use of communications spectra. Intelligence agencies too might be interested in the interception capabilities of single-quanta devices, as multiple such devices working together should be readily able to determine the source of signals. One can also imagine the possibility of stealthy communication capabilities.

\footnotetext{
${ }^{64}$ Gely et al., "Observation and Stabilization of Photonic Fock States in a Hot Radio-Frequency Resonator" (2019).

${ }^{65}$ Meyer et al., "Assessment of Rydberg Atoms for Wideband Electric Field Sensing" (2020); Cox et al., "Quantum-Limited Atomic Receiver in The Electrically Small Regime" (2018).
} 
- Manufacturers in many fields will benefit from advances in materials science from quantum technologies. While quantum computing will help manufacturers design new materials, quantum sensing will allow inspection and characterization of them. Someday, super-precise fabrication may be possible where objects are crafted at the atomic level, making them perfectly matched in size and composition.

\subsubsection{Sensing Gravitational Fields}

Albert Einstein predicted the existence of gravitational waves, but thought them too weak to be sensed. They were, for a time.

Gravitational waves are caused by the acceleration or change of mass through space. This means that you create gravity waves every time your heart beats (or your quartz crystal vibrates). This is similar to the way that a moving charge creates electromagnetic waves, which is the basis of how a radio transmitter works. Gravity is much weaker than electromagnetism, however, and so gravity waves are correspondingly much smaller.

To date, the only gravity waves that we have managed to detect are the waves created as a result of cosmic events that released tremendous amounts of energy - such as the collision of two black holes. Once formed, gravity waves travel at the light speed and pass through our planet (along with eventually everything else in the universe) without much interaction. Almost a hundred years after Einstein's prediction, researchers at the Laser Interferometer Gravitational-Wave Observatory (LIGO), an enormous, ambitious scientific project, made the first direct observation of gravitational waves and of two black holes merging to form a single black hole. ${ }^{66}$ The detection of gravitational waves was accomplished with an interferometer that was able to detect the ever-so-slight compression of space-time in one direction compared with another as a result of the passing gravity wave (see Appendix B for more information).

An interferometer uses a source of light and a beam splitter to send the light in different directions. Mirrors at the end of the arms of the interferometer reflect the light back, where it is superimposed on a sensor. Turn it on and the two beams of light form an interference pattern. If the interference pattern changes, then either the distance between one of the mirrors and the beam splitter must have changed,

${ }_{66}$ Abbott et al., "Observation of Gravitational Waves From a Binary Black Hole Merger" (2016). 
or else something between the mirrors and the beam splitters must have changed the phase of one of the light beams. One application of interferometers is thus making precise measurements of distance and making sure that physical systems stay in calibration.

In the case of the LIGO, the system is designed so that nothing should be able to change the distance between the beam splitter and the mirrors. For starters, all of the optical components are in a vacuum chamber. The devices are built in a region that is not seismically active, and far away from equipment that might cause the ground to vibrate. The idea of the system is that a passing gravity wave literally changes the distance between the splitter and the mirrors. Because gravity waves are directionally aligned, the distance for each mirror changes by a different amount, and the diffraction pattern changes. The longer the arms of the interferometer, the more sensitive the device will be to distortions in the fabric of space-time.

The LIGO interferometer has arms $4 \mathrm{~km}$ long. The beam of light in LIGO is prepared so that by default, if the distance between mirrors does not change, the photodetector senses no light. That is, LIGO harnesses destructive interference as a tool to detect waves. If a gravitational wave is sensed, an arm expands or contracts, thus eliminating the destructive inference and revealing a pattern on the photodetector.

Of course, the interferometer is not perfectly isolated from the ground on which it is built. Trucks drive around, planes pass overhead, and there is always a risk that some stray vibration will also move one of the mirrors. So the LIGO consists of two $4 \mathrm{~km}$ interferometers separated by a great distance. If one senses a change in distance and the other doesn't, that vibration was no gravity wave. But if both sense the same change at the same time, a gravity wave has been detected.

The curvature of the Earth, and other challenges such as the need for seismic stability and a vacuum, limit the size of a terrestrial optical interferometer and thus its sensitivity. A collaboration between the European Space Agency and NASA seeks to build the Laser Interferometer Space Antenna (LISA), which will be formed by three space vehicles separated by 2.5 million $\mathrm{km}$. LISA is expected to be completed in the 2030s. The Chinese Academy of Sciences has a similar project, the TianQin observatory, on a similar timeline, but with 
a target of 3 million km-distant interferometer arms. ${ }^{67}$ Separately, some are proposing the construction of "space-borne gravitational wave detectors based on atom interferometry" that would detect gravity waves acting on collections of perhaps a hundred million atoms falling in the vibration-free environment of space. ${ }^{68}$

LIGO, LISA, and TianQin are all focused on gravitational sensing of the cosmos. What if similar highly accurate sensing technologies based on interferometry were focused on Earth? In the 1990s, the first experiments were conducted that used interferometric synthetic aperture radar (InSAR), an approach that enhances the sensitivity of downward-pointing space-based radar systems by comparing carefully timed radar imagery (interferograms) of the Earth.

In 2002 the GRACE (Gravity Recovery and Climate Experiment) employed twin satellites that orbited roughly $220 \mathrm{~km}$ apart. The GRACE satellites used a microwave ranging system to detect minute changes in the distance between them that are the result of variation in the Earth's gravity field. ${ }^{69}$ The GRACE system is designed to be especially sensitive to changes that result from the collection of water (liquid or solid) on the Earth's surface. As the satellites approach stronger gravity fields, signaling greater concentrations of water, gravity pulls the lead vehicle a little faster and thus increases the distance to the trailing satellite, which itself speeds up a short time later. As the water recedes into the distance, the lead satellite slows down a bit, followed by the second. The GRACE mission produced a monthly, whole planet survey of water, tracking millimeterlevel changes in density. Originally planned for a 5-year mission, the GRACE mission was decommissioned in 2017. ${ }^{70}$ The Gravity Recovery and Climate Experiment Follow-On (GRACE-FO) mission, launched in 2018, uses laser interferometry for increased precision. Also focused on water movement, GRACE-FO will help forecast rising seas and the development of drought.

Optical interferometry has occupied a central place in this discussion, but scientists have also developed cold-atom interferometers to

${ }^{67}$ H.-T. Wang et al., "Science with The TianQin Observatory: Preliminary Results on Massive Black Hole Binaries" (2019).

${ }^{68}$ Loriani et al., "Atomic Source Selection in Space-Borne Gravitational Wave Detection" (2019).

${ }^{69}$ Tapley et al., "The Gravity Recovery and Climate Experiment: Mission Overview and Early Results" (2004).

${ }^{70}$ The European Space Agency operated a similar mission called the Gravity Field and Steady-State Ocean Circulation Explorer (GOCE) from 2009 to 2013. 
sense gravity. ${ }^{71}$ In this approach, two different collections of atoms are initialized in a superposition using lasers; the atoms then interact with gravitational signals, the characteristics of which are revealed in the differences between the two ensembles of atoms. Because the ensembles of atoms themselves are being compared, the ensembles need not be separated by great distances. Indeed, atom interferometers are now miniaturized, with devices resilient enough to be mounted on aircraft and even on small unmanned aerial vehicles (UAVs, also known as drones). ${ }^{72}$ These devices are not sensitive enough for detecting gravity waves, but they are just fine for measuring the Earth's gravity. Miniaturized gravimeters were still research curiosities just a decade ago; today they are available from companies such as AO Sense, Inc.

As gravimetric detection improves in resolution, one might imagine strategic uses of the data collected. With GRACE-FO, the US is sharing with the world information about water and drought. Such predictions inherently have strategic implications, given the likelihood of conflicts resulting from climate change. (For example, the so-called Arab Spring of 2010 and 2011 was driven in part by high food prices attributed to that year's poor crop yields, a likely result of climate change.)

\subsubsection{Quantum Illumination}

In quantum illumination, ${ }^{73}$ entanglement can discern between reflected light and noise, or be used as a kind of object detector. In experimental systems, entangled photons are generated. One of the pair is sent out to the environment while the other is measured. As photons are received in a detector, the measured, entangled photon is compared to received ones to see if it is thermal noise or a reflected photon.

\section{Ghost Imaging}

In ghost imaging, which has both classical and quantum methods, entangled photons are used to sense objects that are not "in view" of a camera.

${ }^{71}$ Bongs et al., "Taking Atom Interferometric Quantum Sensors From The Laboratory to Real-World Applications" (2019).

${ }^{72}$ Weiner et al., "A Flight Capable Atomic Gravity Gradiometer With a Single Laser" (2020).

${ }^{73}$ Quantum illumination, as defined here, goes by several names, including correlated-photon imaging and two-photon approaches. 


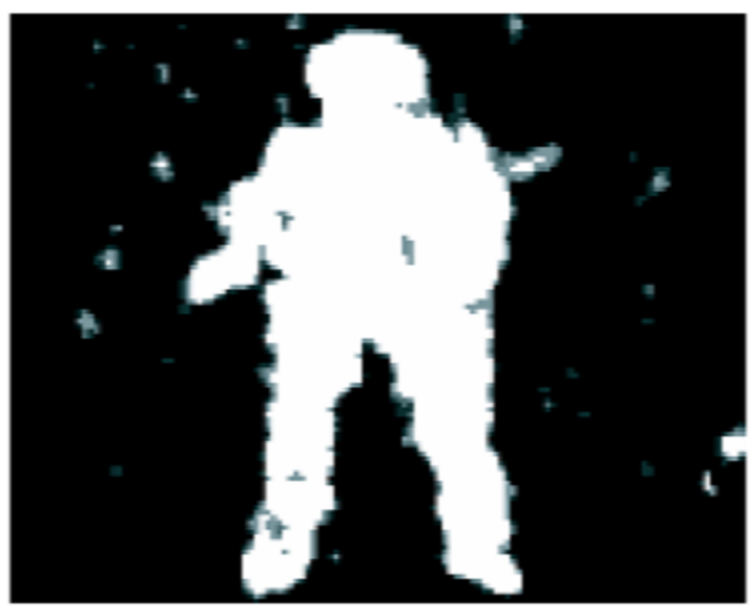

Figure 2.9. Carefully counting scattered and reflected photons from this toy soldier created a "ghost image" of it - an image constructed of an object that was outside the view of a camera. Courtesy of Office of the Secretary of Defense Public Affairs.

In an exciting demonstration of this approach, researchers at the Army Research Laboratory (ARL) published a paper that indirectly imaged a toy soldier (see Figure 2.9). The image was generated using a split beam of light. One beam was directed to the toy soldier, illuminating it. The soldier reflected and scattered photons from the first beam, some of which were collected by a nearby "bucket" detector, a special type of single-photon sensor that, like a water pail, collects photons without mapping out their specific location. The second beam was directed into a CCD camera. A separate system correlated the photons between the bucket detector and CCD to reveal which light was reflected and which was scattered. The resulting image is clearly of a toy soldier. The approach works on all wavelengths, meaning that shining different frequency light could reveal chemical composition of an object (perhaps revealing it to be real or a decoy). ${ }^{74}$

The ARL scientists built on this achievement with a demonstration of how ghost imaging could be applied to challenges in satellitebased sensing, and sensing in other difficult conditions, including underwater. In a follow-up study, the ARL team introduced "turbulence" to the setup by adding a $550{ }^{\circ} \mathrm{C}$ heater. Despite the turbu-

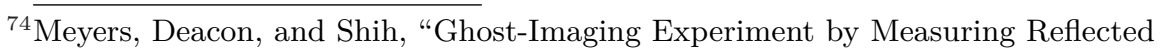
Photons" (2008). 

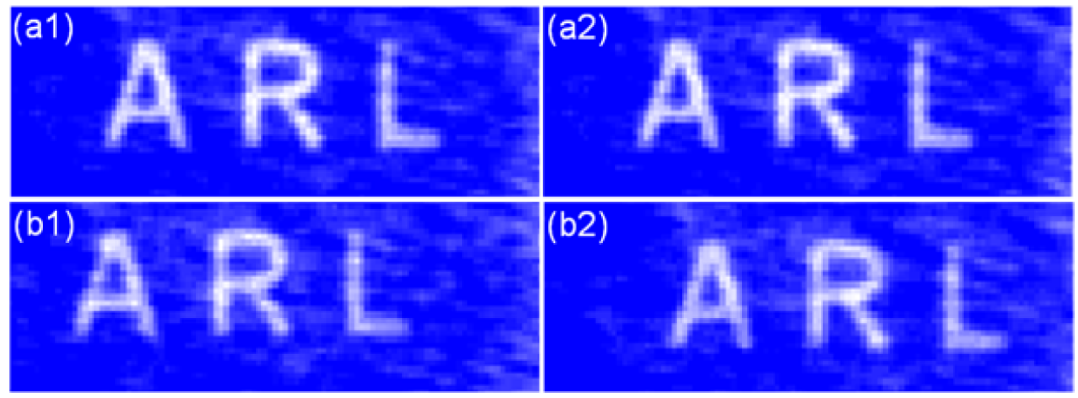

Figure 2.10. The ghost images in panels a1 and a2 were captured under $550{ }^{\circ} \mathrm{C}$ turbulence. In frames b1 and b2, the experimenters correlated photons captured at different times, also under turbulence. In b1, the image is based on photons five frames before the other detector; in b2, five frames after. Figure CC-BY Meyers and Deacon (2015).

lence, one can make out the letters A R L in their demonstration. The ARL's advances in ghost imaging could make it possible to see clearly on chaotic, turbulent, hot, and smoky battlefields. Another demonstration used cloudy water as the "turbulence." Nevertheless, the ARL's "A" is relatively readable, elucidating implications for underwater detection (light is absorbed by water, thus limiting sensing distance). Subsequently, ARL showed that it could image objects with photons measured at different times (see Figure 2.10). This demonstration is important, because it signals the potential to use ghost imaging for moving objects. ${ }^{75}$

The ARL techniques would be useful for many civilian contexts. Vehicle safety systems might use indirect evidence from "unseen" vehicles around corners or difficult-to-see pedestrians based upon how light reflects and scatters around them. Scientists are also excited about ghost imaging's potential to contribute to image compression and to multi-spectral analysis. Scientists at the Brookhaven National Laboratory announced in 2020 that they intended to use ghost imaging in an X-ray microscope to take advantage of radiation avoidance from a split beam of light. That is, X-rays damage many study samples, thus the scientists will try to use ghost imaging to reduce the amount of X-ray exposure to the sample while using correlated photons to maintain high resolution (see Figure 2.11).

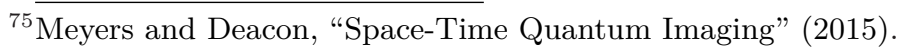




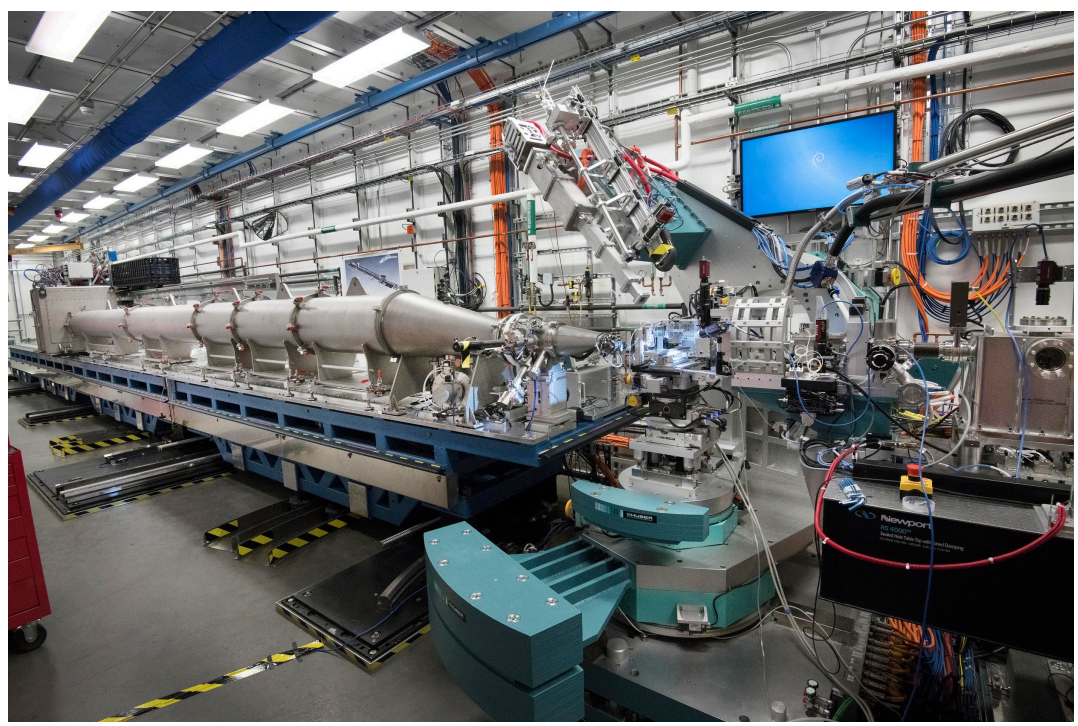

Figure 2.11. Scientists at Brookhaven National Laboratory are constructing a microscope that will use ghost imaging to minimize the harm to samples under examination. The idea is to split and entangle the $\mathrm{X}$-ray photons, and send just a portion of the harmful X-rays to the sample. Because the photon beams are correlated, it should be possible to infer data from the photons that never interact with the sample. The goal is to achieve high resolution without exposing the sample to the full, damaging effects of X-rays. Courtesy of Brookhaven National Laboratory.

\subsubsection{Quantum Radar}

Quantum illumination is a candidate sensing approach for expanding the sensitivity of military radar systems ${ }^{76}$ and to make radar systems themselves more difficult to detect. ${ }^{77}$

Quantum radar involves generating billions of entangled photon pairs to illuminate targets. One photon from the pair, the signal photon, is sent to the environment in hopes it will hit a target and be reflected back to the radar array. The other photon, the idler or ancilla photon, is retained in memory (see Figure 2.13). Photons received by the array are then compared to the retained idler photons, where the operator can determine whether those received photons are correlated or not. Non-correlated photons are noise from the atmosphere, but correlated ones reveal information about the reflective object.

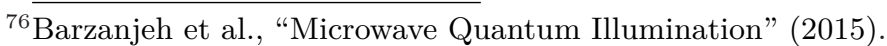

${ }^{77}$ Marco Lanzagorta, Quantum Radar (2011). 


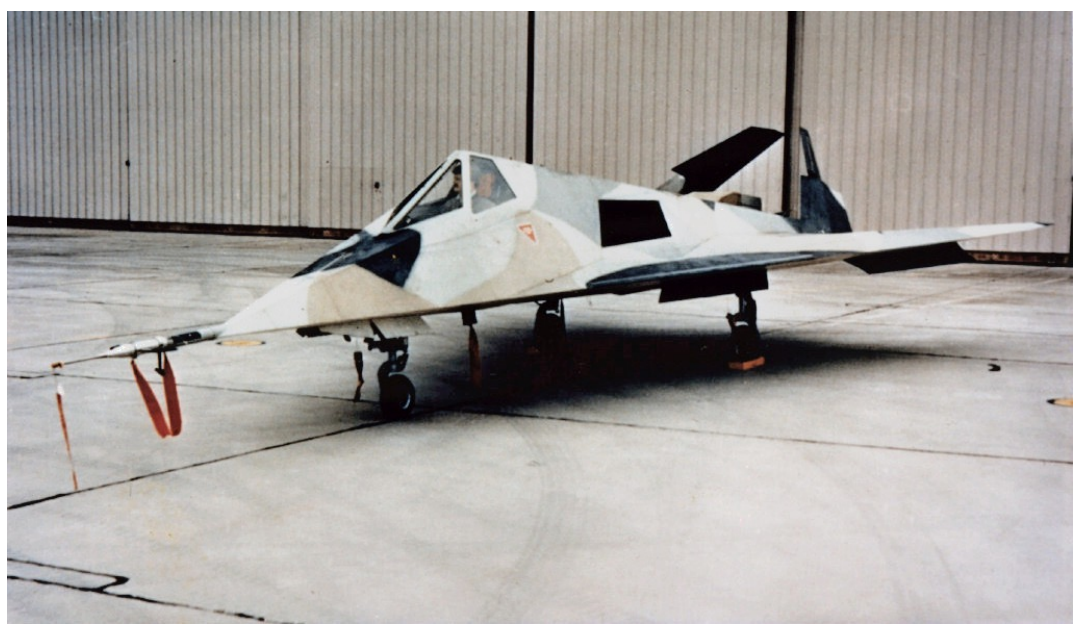

Figure 2.12. Lockheed Skunk Works' HAVE BLUE brought about a revolution in low-observable "stealth" aircraft, and minted billions for Lockheed, Silicon Valley's largest employer until the internet revolution in the 1990s. Quantum sensing imperils stealth technologies. Photo public domain DARPA.

To an adversary, those billions of photons are simply atmospheric noise. Thus quantum sensing is "stealthy"; the idler photon allows the operator to distinguish between background noise while focusing attention on the quantum radar signal, those reflected signal photons that correlate with the idler photons.

The military applications of such quantum illumination for radar are many. A photonic approach should detect low-observable objects, such as vehicles that use "stealth" technology (see Figure 2.12) or even forms of electromagnetic jamming. For instance, one use foreseen by the Air Force is to use quantum technology to counter "digital radio frequency memory jamming," a technique where an enemy fighter captures emitted radar pulses and replays them at a different speed in order to confuse air defense systems. Adversaries might try to jam quantum radar by sending billions of noise photons into the array. However, if quantum radar works properly, the operator can simply filter out those noise photons based on correlations between the desired signal and idler photons. Militaries might also use these techniques for navigation. A submarine, for instance, could use reflected photons to sense undersea dangers, such as mines. ${ }^{78}$

${ }^{78}$ Marco Lanzagorta, Jeffrey Uhlmann, and Salvador E. Venegas-Andraca, "Quantum Sensing in The Maritime Environment" (2015). 


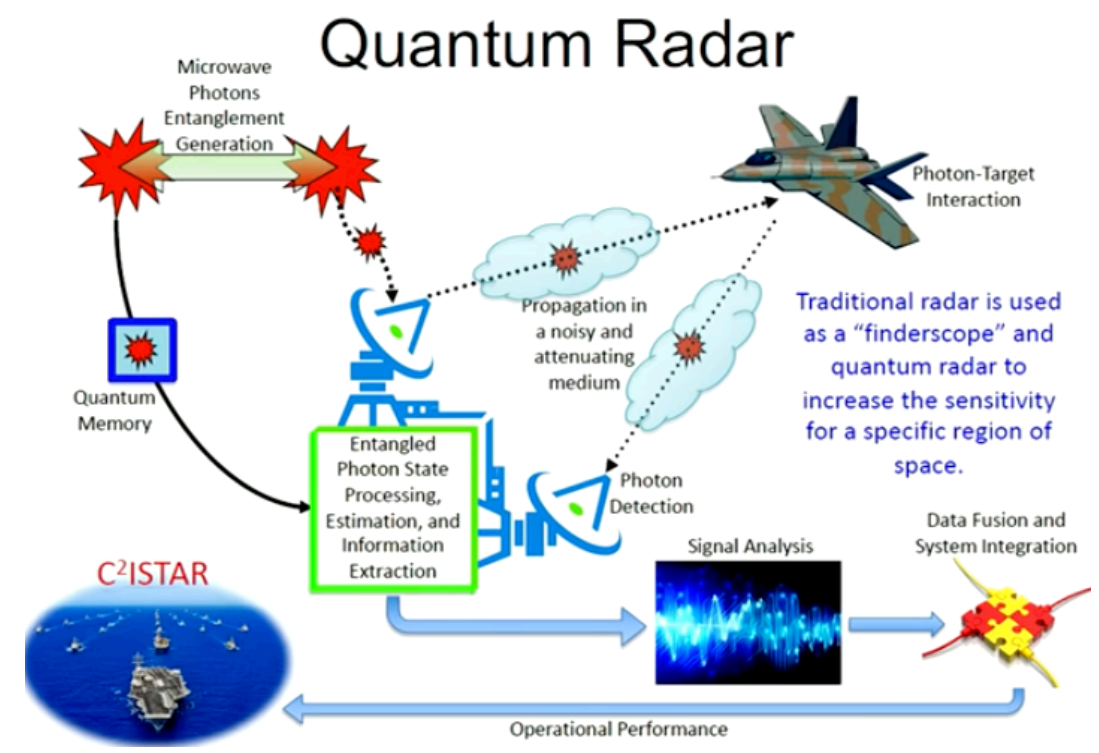

Figure 2.13. In a 2018 address to the National Academies, Dr. Marco Lanzagorta, explained how quantum radar might detect low-observable aircraft. Image courtesy US Naval Research Laboratory.

There are significant engineering challenges to quantum radar; ${ }^{79}$ however, theoreticians believe these are surmountable, and recent developments suggest alternative approaches that could produce working quantum radar. The main challenges surround generation and entanglement of billions of photons and the need to have some form of quantum memory to retain idler photons for comparison to signal photons. But in both challenges, there are reasons to believe that innovations or different approaches could make quantum radar possible. ${ }^{80}$ With respect to entangled photons, great strides have been made in recent decades in lasers, a key complementary technology for quantum innovation. Investment in laser technology is bound to continue and expand, because of lasers' importance to quantum sensing, computing, and communications (see Figure 2.1 and Figure 2.3). Because radar systems typically operate in the microwave band, research funding for photonic generation at microwave frequencies may

${ }^{79}$ Cho, "The Short, Strange Life of Quantum Radar" (2020).

${ }^{80}$ Marco Lanzagorta and Jeffrey Uhlmann, "Opportunities and Challenges of Quantum Radar" (2020). 
be a signpost of quantum radar programs. Separately, there have also been advances in quantum memory; however, an alternative approach to quantum radar may eliminate the memory requirement entirely. Researchers at University of Waterloo proposed a protocol that measures the idler photon immediately, thus allowing the signal photon to be compared at some later time using classical memory. ${ }^{81}$

Quantum radar has applications in outer space, which makes sense because there is less photonic attenuation in space than in a planet's atmosphere. A satellite equipped with a quantum radar system might be used for a range of applications: for detecting ballistic missiles, discovering adversaries' secret satellites, and even finding dangerous space junk. ${ }^{82}$

\subsection{From SIGINT to MASINT}

Some quantum technologies discussed in this chapter raise few unmanageable policy issues, in part because with some of these technologies, the subject would know they were being measured. The individual would have to be in a Faraday-caged room so their body could be isolated from sensor-befuddling power lines, radio waves and the like. The individual would also have to remain extremely still until technologies catch up to track moving objects. However, other quantum metrology and sensing approaches have characteristics of remote sensing. That is, like many surveillance technologies, they can be used against unwilling or unknowing subjects, raising policy issues ranging from individual privacy to national security concerns. The primary dividing line is between magnetic field sensing and gravitational sensing. Gravitational sensing can be made extremely sensitive, and because gravity cannot be shielded, countermeasures are limited.

The emergence of deployable and highly precise gravitational sensors could cause a shift in intelligence gathering. In recent decades, the power of signals intelligence (SIGINT) (Section 7.2 (p. 264)) has astonished many. Signals intelligence focuses on communications and radar systems and is the primary responsibility of the National Security Agency (NSA) in the United States. By monitoring fiber-optic and other forms of communications, many nations have surprising powers to track people, identify them, and to listen in to their con-

${ }^{81}$ C. W. Chang et al., "Quantum-Enhanced Noise Radar" (2019).

${ }^{82}$ Marco Lanzagorta and Jeffrey Uhlmann, "Space-Based Quantum Sensing for LowPower Detection of Small Targets" (2015). 
versations. In recent decades, the NSA has attracted respect and resentment as its surprising and strong capabilities have been brought to bear in conflicts. The NSA is thought to be the largest employer of mathematicians in the world; ${ }^{83}$ it has emerged as a central asset with the rise of computing and the need to both secure computers and to attack them in surprising ways.

Quantum technologies, for reasons explained in Chapter 7, may secure more communications and make metadata surveillance impossible, thus frustrating SIGINT efforts. But at the same time, quantum sensing technologies will give governments more power to engage in MASINT, measurement and signature intelligence. MASINT approaches focus on the measurement of objects and their "signatures." This includes what objects are, whether they are moving, and whether they have been used recently. For instance, by sensing attributes of an armored fighting vehicle, one might identify it, understand whether it is vibrating or moving, and by studying heat dissipation, whether and how recently the tank gun has been fired. As quantum sensing comes into use, a trio of different agencies will become more important: the Defense Intelligence Agency (DIA), the National Reconnaissance Office (NRO), and the National Geospatial-Intelligence Agency (NGA). Companies that foresee the shift to MASINT, such as ColdQuanta, are already collecting strategic board members with experience at these agencies.

\subsection{Quantum Sensing: Conclusion}

This long chapter foreshadows a key finding of this book: that quantum sensing is likely to be the most consequential of quantum technologies. As the technologies discussed in this chapter transition from the laboratory to the marketplace, quantum metrology and sensing have the potential to alter how nations monitor and engage in conflict. Since the deployment of GPS in the 1990s, quantum measuring technologies have provided the US military with incremental advantage in conflict. Such capabilities are both increasing and becoming more widely available. Some quantum sensors and components are commercially available.

As impressive as the military and intelligence applications are, quantum sensing could also contribute to drug development, medical diagnostics, medical devices including prosthetics linked to the brain,

${ }^{83}$ M. Wagner, "The Inside Scoop on Mathematics at The NSA" (2006). 
and more efficient and targeted mineral extraction. Quantum sensors are also a precursor for quantum computers and communications.

The next three chapters build on quantum sensing by explaining the history of quantum computing, its likely uses, and the current landscape of the field. 